NASA/CR-1/998- 207518

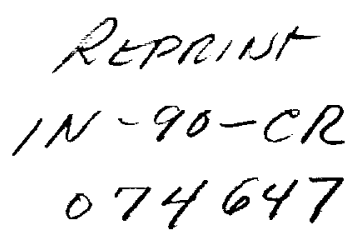

Deuterated Water in Comet C/1996 B2 (Hyakutake) and its Implications for the Origin of Comets

D. Bockelée-Morvan, D. Gautier, D.C. Lis, K. Young, J. Keene, T.G. Phillips, T. Owen, J. Crovisier. P.F. Goldsmith, E.A. Bergin, D. Despois, \& A. Wootten

1998-5

Accepted for publication in

Icarus 


\section{Abstract}

The close approach to the Earth of comet C/1996 B2 (Hyakutake) in March 1996 allowed searches for minor volatile species outgassing from the nucleus. We report the detection of deuterated water (HDO) through its $1_{01}-0_{00}$ rotational transition at 464.925 $\mathrm{GHz}$ using the Caltech Submillimeter Observatory. We also present negative results of a sensitive search for the $J(5-4)$ line of deuterated hydrogen cyanide (DCN) at $362.046 \mathrm{GHz}$.

Simultaneous observations of two rotational lines of methanol together with HDO in the same spectrum allow us to determine the average gas temperature within the telescope beam to be $69 \pm 10 \mathrm{~K}$. We are thus able to constrain the excitation conditions in the inner coma and determine reliably the HDO production rate as $(1.20 \pm 0.28) \times 10^{26} \mathrm{~s}^{-1}$ on March 23-24, 1996. Available IR, UV and radio measurements lead to a water production rate of $(2.1 \pm 0.5) \times 10^{29} \mathrm{~s}^{-1}$ at the time of our HDO observations. The resulting $\mathrm{D} / \mathrm{H}$ ratio in cometary water is thus $(29 \pm 10) \times 10^{-5}$, in good agreement with the values of $\left(30.8_{-5.3}^{+3.8}\right) \times 10^{-5}$ (Balsiger et al. 1995) and $(31.6 \pm 3.4) \times 10^{-5}$ (Eberhardt et al. 1995) determined in comet $\mathrm{P} /$ Halley from in situ ion mass spectra. The inferred $3 \sigma$ upper limit for the $\mathrm{D} / \mathrm{H}$ ratio in $\mathrm{HCN}$ is $1 \%$.

Deuterium abundance is a key parameter for studying the origin and the early evolution of the Solar System and of its individual bodies. Our HDO measurement confirms that, in cometary water, deuterium is enriched by a factor of at least 10 relative to the protosolar ratio, namely the $\mathrm{D} / \mathrm{H}$ ratio in $\mathrm{H}_{2}$ in the primitive Solar Nebula which formed from the collapse of the protosolar cloud. This indicates that cometary water has preserved a major part of the high $\mathrm{D} / \mathrm{H}$ ratio acquired in this protosolar cloud through ionmolecule isotopic exchanges or grain-surface reactions and was not re-equilibrated with $\mathrm{H}_{2}$ in the Solar Nebula. However, there are strong presumptions that interstellar grains were partly mixed in the early nebula prior to cometary formation with water reprocessed in the warm inner part of the nebula and transported by turbulent diffusion. Scenarios of formation of comets consistent with these results are discussed.

Subject headings: comets, deuterium, Solar Nebula, submillimeter spectroscopy

\section{Introduction}

Deuterium is a sensitive indicator of the origin of water ice in comets. Cometary ices may have condensed in the primitive Solar Nebula or may have come directly from the protosolar cloud from which the nebula was formed. In both cases, deuterium fractionation must have occurred at low temperatures between molecular hydrogen (the main reservoir of deuterium) and water, so the $\mathrm{D} / \mathrm{H}$ ratio in $\mathrm{H}_{2} \mathrm{O}$ should be higher than the $\mathrm{D} / \mathrm{H}$ ratio in $\mathrm{H}_{2}$ in the Solar Nebula (usually reported as the protosolar ratio), estimated to be $\sim 3 \times 10^{-5}$ (see Section 4.2).

The difference between the two scenarios comes from the kinetics of isotopic reactions: in cold dense interstellar clouds, the fractionation occurs through ion-molecule reactions and grain-surface chemistry and is very fast, so that the equilibrium can be reached even at a low temperature, resulting in high $\mathrm{D} / \mathrm{H}$ enrichment factors in deuterated species (Watson 1974; Irvine and Knacke 1989). By contrast, the deuterium fractionation in the Solar Nebula occurs through reactions between neutral species (e.g. Geiss and Reeves 1981). In this case, the rate of isotopic exchange depends on temperature, pressure and time. It goes to zero at the low temperature and pressure values expected in the outer part of the nebula. In addition, the fractionation stops when the hydrogen escapes from the nebula. Therefore, a deuterium enrichment higher than a factor of 3 with respect to the protosolar value is not expected in the Solar Nebula.

In summary, a high (much higher than 3 ) $\mathrm{D} / \mathrm{H}$ enrichment in cometary water would thus be consistent with an interstellar origin of the ices from which these objects formed. On the other hand, a low $\mathrm{D} / \mathrm{H}$ ratio (close to the protosolar ratio) would be consistent with a turbulent and well mixed nebula, at least out to the region of cometary formation.

Until recently, the $\mathrm{D} / \mathrm{H}$ ratio measured in the water of $\mathrm{P} /$ Halley was too uncertain to permit firm conclusions (Eberhardt et al. 1987). However, the results from the mass spectrometers aboard Giotto have been improved. The detailed analysis of the data of the ion mass spectrometer (IMS) by Balsiger et al. (1995) and those of the neutral mass spectrometer (NMS) by Eberhardt et al. (1995) independently lead the two groups to precise and consistent estimates of $\mathrm{D} / \mathrm{H}$ in water of $\left(30.8_{-5.3}^{+3.8}\right) \times 10^{-5}$ (IMS) and $(31.6 \pm 3.4) \times 10^{-5}$ (NMS). Such a high value implies an interstellar origin for at least a substan- 
tial part of the $\mathrm{P} /$ Halley material. However, it might be argued that this comet may be atypical and not representative of most comets. It is thus valuable to measure the $\mathrm{D} / \mathrm{H}$ ratio in other comets.

The apparition of the bright comet $\mathrm{C} / \overline{1} \overline{9} \overline{\mathrm{6}} \mathrm{B} 2$ Hyakutake provided an opportunity to detect HDO from the ground by means of heterodyne spectroscopy at submillimeter wavelengths. Indeed, soon after its discovery on January 30, 1996 (Nakamura and Nakano 1996), this long-period comet turned out to be an intrinsically bright comet destined to make a very close approach to Earth on March 25, 1996 at only 0.102 AU (Marsden 1996). Due to the excellent viewing geometry, this comet proved to be an ideal and unprecedented target. Observations of the HDO $1_{01}-0_{00}$ line at $464.925 \mathrm{GHz}$ were organized on short notice at the Caltech Submillimeter Observatory (CSO). Good atmospheric conditions allowed an unambiguous detection (Lis et al. 1996a). This is the first detection of cometary HDO from the ground. In addition a sensitive search for DCN was performed.

Section 2 describes the observations. They are part of the overall CSO observational campaign for comet C/1996 B2 (Hyakutake) presented by Lis et al. (1997). In Section 3, the HDO production rate and $\mathrm{HDO} / \mathrm{H}_{2} \mathrm{O}$ abundance ratio are derived. Section 4 discusses the $\mathrm{D} / \mathrm{H}$ ratio and its implications for the origin of comets and the structure of the primitive Solar Nebula. The excitation model for HDO is presented in Appendix A.

\section{Observations}

Observations of HDO and DCN in comet C/1996 B2 (Hyakutake) were carried out using the $10.4-\mathrm{m}$ Leighton telescope of the Caltech Submillimeter Observatory located on Mauna-Kea, Hawaii. A log of the observations is given in Table 1. Other CSO observations of comet Hyakutake are presented by Lis et al. (1997).

The $1_{01}-0_{00}$ line of HDO at $464.92452 \mathrm{GHz}$ (DeLucia et al. 1971) was observed on March 23.5 and 24.5 $1996 \mathrm{UT}$, when the comet was at 0.11-0.12 AU from the Earth and 1.1 AU from the Sun. The observations were performed near meridian transit when the comet was at $60-80^{\circ}$ elevation. Observing conditions were quite good, characterized by zenith opacities at $225 \mathrm{GHz}$ near $0.05-0.06$, as measured by a tipping radiometer. The single sideband (SSB) system temperature referred to above the Earth's atmosphere at the elevation of the comet was about $1500 \mathrm{~K}$ at 465 $\mathrm{GHz}$.

Observations of the $J(5-4)$ transition of DCN at $362.04648 \mathrm{GHz}$ (Pickett et al. 1996) were carried out on March 26.5, 1996 UT. The data were taken under good weather conditions characterized by a $225 \mathrm{GHz}$ zenith optical depth of 0.06-0.07. However, the SSB system temperature was relatively high $(\sim 1200 \mathrm{~K})$ due to the low elevation of the comet $\left(27-30^{\circ}\right)$.

Comet Hyakutake was tracked using an ephemeris kindly supplied by Don Yeomans (private communication). The ephemeris used on March 23 and 24 is based upon orbital elements referred to as Yeomans" "Solution 18" which were computed from 354 astrometric positions obtained from Jan. 1 to Mar. 21, 1996. "Solution 21" was used during the DCN observation on March 26. Ephemerides and pointing were checked frequently by performing five-point maps of HCN transitions and found to be accurate to $\sim 3^{\prime \prime}$ r.m.s.

The data of March 24 and 26 were acquired in the beam-switching mode, by chopping the secondary mirror at a $0.8 \mathrm{~Hz}$ rate with a throw of $4^{\prime}$ in azimuth. Those of March 23 were made in position-switching mode, with the reference position offset by $10^{\prime}$. The observations were made using the facility SIS receivers operating in a double-sideband (DSB) mode with signal and image bands separated by $3 \mathrm{GHz}$. In order to minimize the system temperature, the receivers were tuned to the HDO and DCN frequencies in the upper sidebands. We used simultaneously two 1024-channel acousto-optical spectrometers (AOS) as backends: a low-resolution AOS which covers a total bandwidth of $560 \mathrm{MHz}$ with an effective spectral resolution of $\sim 1$ $\mathrm{MHz}\left(0.6 \mathrm{~km} \mathrm{~s}^{-1}\right.$ at $\left.465 \mathrm{GHz}\right)$ and a high-resolution AOS of $50 \mathrm{MHz}$ bandwidth providing a spectral resolution of $\sim 100 \mathrm{kHz}\left(0.06 \mathrm{~km} \mathrm{~s}^{-1}\right.$ at $\left.465 \mathrm{GHz}\right)$.

The data were calibrated using the standard chopper-wheel method to give antenna temperatures outside the atmosphere. The intensity calibration was checked by observing IRC +10216 . The calibration uncertainty is typically $10 \%$ for lines observed with the $50 \mathrm{MHz}$ spectrometer. For the interpretation of the observed signals, antenna temperatures were converted into main beam brightness temperatures using the relation $T_{B}=T_{A}^{*} / \eta_{\mathrm{mb}}$, with efficiencies $\eta_{\mathrm{mb}}$ measured on Jupiter (Table 2). The telescope beam size (HPBW) at the distance of the comet corresponded to approximately $1200 \mathrm{~km}$ for HDO observations and $1600 \mathrm{~km}$ for DCN observations. 


\title{
Deuterated Water in Comet C/1996 B2 (Hyakutake) and its Implications for the Origin of Comets
}

\author{
D. Bockelée-Morvan, D. Gautier \\ Observatoire de Paris, F-92195, Meudon Principal Cedex, France \\ D.C. Lis, K. Young, J. Keene, T.G. Phillips \\ Caltech Submillimeter Observatory, MS 320-47, Pasadena, CA 91125, USA \\ T. Owen \\ Institute for Astronomy, University of Hawaii, 2680 Woodlawn Drive, Honolulu, Hawaii 96822, USA \\ J. Crovisier \\ Observatoire de Paris, F-92195 Meudon Principal Cedex, France \\ P.F. Goldsmith \\ NAIC, Cornell University, Ithaca, NY 14853, USA \\ E.A. Bergin \\ Harvard-Smithsonian Center for Astrophysics, MS 66, Cambridge, MA 02138, USA \\ D. Despois \\ Observatoire de Bordeaux, B.P. 89, F-33270, Floirac, France \\ and \\ A. Wootten \\ National Radio Astronomy Observatory, Charlottesville, VA 22903-2475, USA
}

ICARUS 1998, in press 



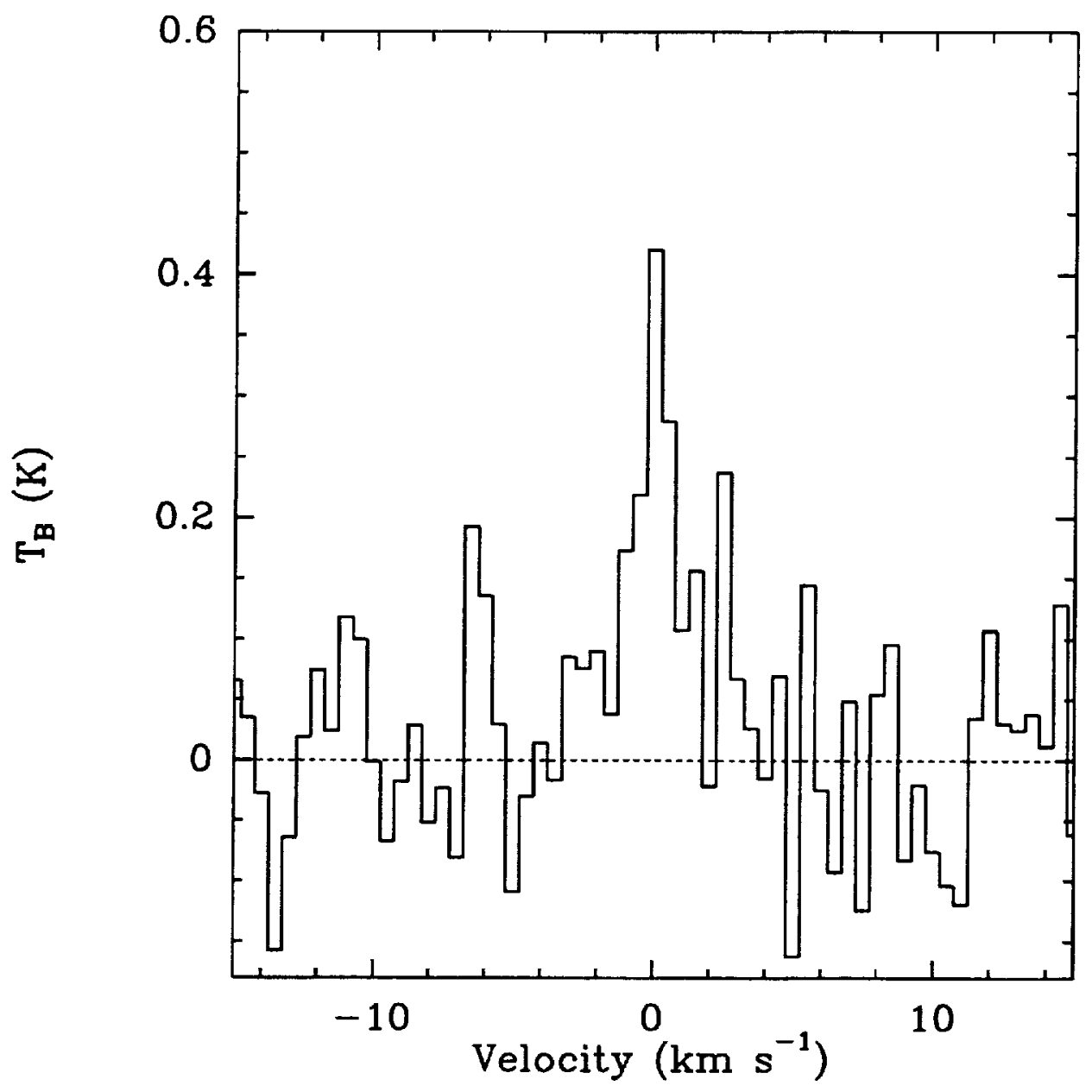

Fig. 1.- The $1_{01}-0_{00}$ line of HDO at $464.925 \mathrm{GHz}$ in C/1996 B2 (Hyakutake) observed on March 23.5-24.5 UT with the $50 \mathrm{MHz}$ spectrometer. The velocity scale is with respect to the comet nucleus. The spectrum has been smoothed to a spectral resolution of $0.5 \mathrm{~km} \mathrm{~s}^{-1}$.

The DCN line was not detected. HDO was detected independently on March 23.5 and $24.5 \mathrm{UT}$ (Table 3). The signal-to-noise ratio is $\sim 7$, when all observations are averaged. Figure 1 shows the average spectrum obtained with the high-resolution spectrometer. The average HDO spectrum acquired with the low-resolution spectrometer is shown in Fig. 2. Two methanol lines are present in the spectrum: the $(9,2)-(9,1) A^{+}-A^{-}$line at $464.83467 \mathrm{GHz}$ and the $(15,0)-(14,1) A^{+}$line at $461.75360 \mathrm{GHz}$ in the image band (frequencies from $\mathrm{Xu}$ and Lovas 1997). The observed integrated line intensities of HDO and DCN are given in Table 3. They were measured on the high-resolution spectra in the -1.5 to $1.5 \mathrm{~km} \mathrm{~s}^{-1}$ velocity interval. Lines of other molecules observed with a high signal-to-noise ratio during the same period were found to be confined to this velocity range ( $\mathrm{Lis}$ et al. 1997).

\section{3. $\mathrm{D} / \mathrm{H}$ ratio in water and hydrogen cyanide}

\subsection{HDO production rate}

The HDO column density $N_{\text {HDO }}$ is related to the observed line intensity integrated over velocity $\int T_{B} d v$ by:

$$
\int T_{B} d v=\frac{h c^{3}}{8 \pi k \nu_{u l}^{2}} A_{u l} f_{u} N_{\mathrm{HDO}}
$$

where $A_{u l}$ is the Einstein $A$-coefficient of the transition, $\nu_{u l}$ is the rest frequency, and $f_{u}$ is the fractional population of the upper level. Eq.(1) assumes that the emission is optically thin and that the excitation conditions do not vary significantly within the region 


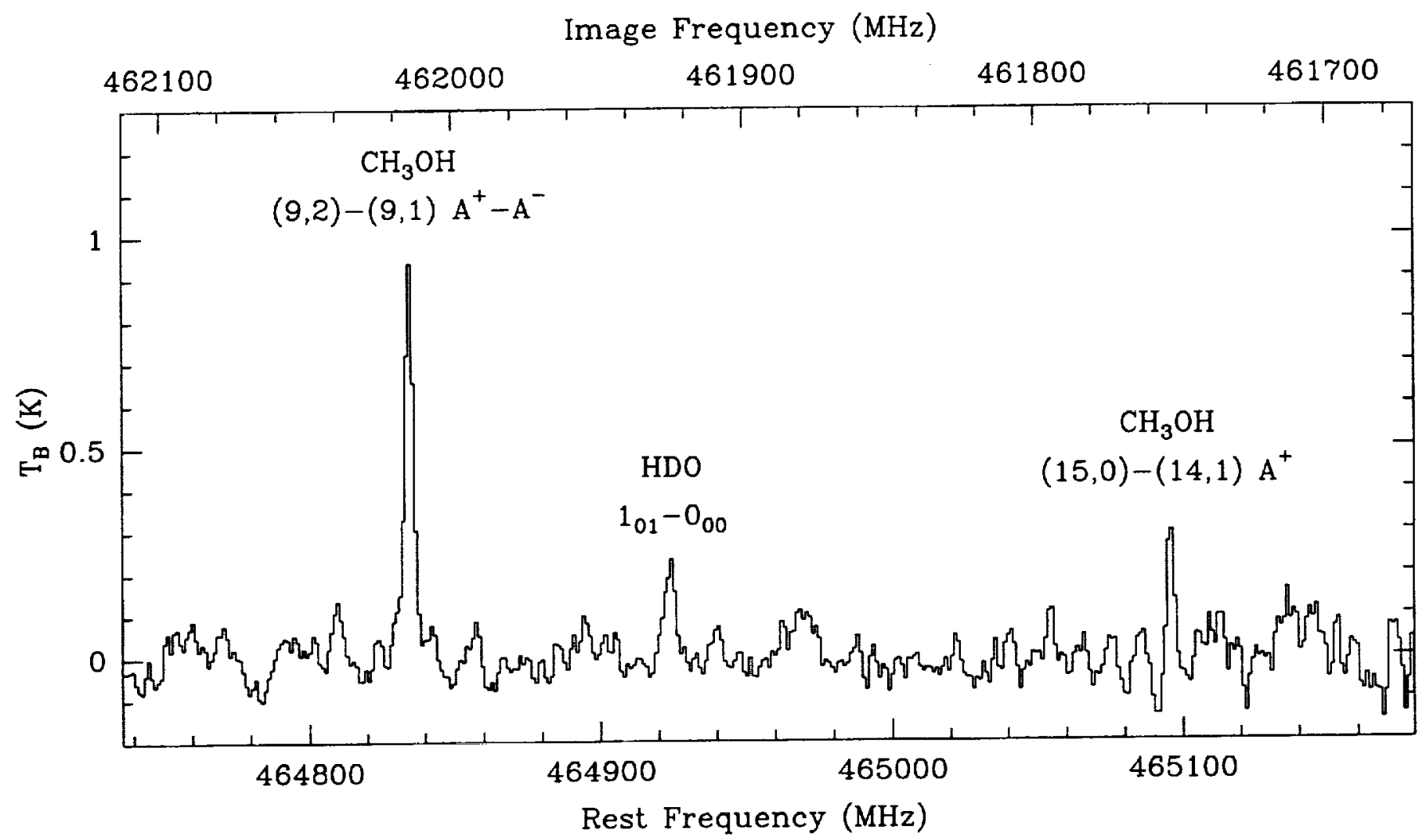

Fig. 2. - Spectrum of the $1_{01}-0_{00}$ line of $\mathrm{HDO}$ at $464.925 \mathrm{GHz}$ in $\mathrm{C} / 1996 \mathrm{~B} 2$ (Hyakutake) observed on March 23.5-24.5 UT with the $560 \mathrm{MHz}$ spectrometer. Two $\mathrm{CH}_{3} \mathrm{OH}$ lines are present in the spectrum: the $(9,2)-(9,1)$ $A^{+}-A^{-}$and the $(15,0)-(14,1) A^{+}$lines at $464.8347 \mathrm{GHz}$ and $461.7536 \mathrm{GHz}$, respectively. The spectral resolution is $1.08 \mathrm{MHz}\left(0.7 \mathrm{~km} \mathrm{~s}^{-1}\right)$.

sampled by the beam.

As shown in Appendix $\mathrm{A}$ and in Fig. 5, with a beam size of $1200 \mathrm{~km}$ in diameter, the observations are sensitive to the collisional region at thermal equilibrium (LTE), where the population of rotational levels is described by the Boltzmann distribution:

$$
f_{u}=\frac{g_{u}}{Z\left(T_{k i n}\right)} e^{-\frac{E_{u}}{k T_{k i n}}}
$$

where $g_{u}$ and $E_{u}$ are the statistical weight and the energy of the upper level, respectively, $T_{k i n}$ is the gas kinetic temperature, and $Z\left(T_{k i n}\right)$ is the partition function. In a first approximation, it is thus relatively straightforward to infer the HDO column density from the observed line intensity, as long as the gas kinetic temperature can be reliably determined.

According to hydrodynamical models of cometary comae, the gas kinetic temperature is expected to vary as a function of the distance from the nucleus. The HDO molecules contributing most $(>80 \%)$ of the observed signal are situated within 100 to 2000 $\mathrm{km}$ from the nucleus. The gas temperature given by theoretical calculations is not reliable enough, due to the number and complexity of heating and cooling processes, so direct temperature measurements have to be used. The two methanol lines present in the HDO spectrum (Fig. 2) allow a direct estimation of $T_{k i n}$ within the field of view. Their relative intensities are consistent with a $\mathrm{CH}_{3} \mathrm{OH}$ rotational temperature of $69 \pm 10 \mathrm{~K}$ (Lis et al. 1997). Other temperature 
measurements are also available (Lis et al. 1997; Biver 1997) and are summarized in Table 4. They all agree with a rotational temperature of about $70 \mathrm{~K}$. There is no significant variation of the rotational temperature with beam size. Calculations with a full excitation model (Bockelée-Morvan et al. 1994; Biver 1997) show that $\mathrm{CO}$ and $\mathrm{CH}_{3} \mathrm{OH}$ are close to LTE in the sampled region, so that their rotational temperature follows the kinetic temperature of the gas. Adopting a kinetic temperature of $69 \pm 10 \mathrm{~K}$ for interpreting the HDO observations is thus secure. In the LTE approximation, an uncertainty of $10 \mathrm{~K}$ in the assumed temperature affects the population of the $l_{01}$ rotational level and the inferred HDO column density and production rate by $\sim 16 \%$.

For a more precise evaluation, we have to take into account radiative decay and infrared pumping, which affect the average population of the $1_{01}$ rotational level. Using the excitation model presented in Appendix A, we found that the upper level population within the CSO beam is increased by $30-40 \%$ compared to that inferred from the LTE model at 69 $K$, depending whether collisions with electrons are included in the computations or not. However, the population of the $1_{01}$ level in the full excitation model is less sensitive to the temperature: a $10 \mathrm{~K}$ temperature variation affects the population by only $\sim 11 \%$.

The HDO column density deduced from the comprehensive excitation model is $(9.2 \pm 1.9) \times 10^{12}$ $\mathrm{cm}^{-2}$ for March 23-24. In order to convert the column density into a HDO production rate, we assumed a Haser density distribution. A more realistic angular density distribution is not required. Numerical simulations show that production rates calculated in cases of strongly anisotropic patterns do not differ by more than $20 \%$ from those calculated in the isotropic approximation (Biver 1997; Biver et al. 1997). In the case of comet Hyakutake, the HDO line (Fig. 1) as well as lines of other molecules detected with high signal-to-noise ratios at CSO present only moderate velocity shifts (Lis et al. 1997). Maps of the HCN coma also suggest a roughly isotropic gaseous coma, although some deviations from spherical symmetry are present (Lis et al. 1996b). Thus, we do not expect our HDO production rate calculations to be significantly affected by the isotropic approximation.

Following Crovisier (1994), the photodissociation rate was taken to be $1.3 \times 10^{-5} r_{h}^{-2} \mathrm{~s}^{-1}$, where $r_{h}$ is the heliocentric distance. The HDO production rate is only weakly sensitive to this parameter. We as- sumed an expansion velocity of $0.75 \mathrm{~km} \mathrm{~s}^{-1}$. This outflow velocity is consistent with the shapes of the radio lines observed in $\mathrm{C} / 1996 \mathrm{~B} 2$ (Hyakutake) at the end of March 1996 (Lis et al. 1997; Biver 1997). The inferred HDO production rate scales proportionally to the assumed outflow velocity, so that a velocity $10 \%$ higher would result in a HDO production rate $10 \%$ higher.

Finally, we tested the sensitivity of the derived production rate to pointing errors. The effect is rather small. An offset of $5^{\prime \prime}$ in the pointing (those effectively observed during pointing checks were $3-5^{\prime \prime}$ at most) would lead to underestimating the HDO production rate by $10 \%$.

In Table 5, we give the $1 \sigma$ confidence limit in the HDO production rate, taking into account the statistical uncertainty along with all the uncertainties discussed above.

\subsection{Deuterium abundance in water}

To determine with good confidence the $\mathrm{D} / \mathrm{H}$ ratio in water $(\mathrm{D} / \mathrm{H})_{\mathrm{H}_{2} \mathrm{O}}$, the water production rate at the time of the HDO observations is needed. Table 5 summarizes determinations available from different techniques. Ironically, the $\mathrm{H}_{2} \mathrm{O}$ production rate seems not as well constrained by observations as the production rate of HDO.

Water was directly observed on March 24.5 through several ro-vibrational lines in the $\nu_{1}+\nu_{2}+\nu_{3}-\nu_{1}$ vibrational band at $5084 \mathrm{~cm}^{-1}$ using the NASA Infrared Telescope Facility (IRTF; Mumma et al. 1996). A production rate $Q_{\mathrm{H}_{2} \mathrm{O}}=(2.3 \pm 0.3) \times 10^{29} \mathrm{~s}^{-1}$ was derived (Dello Russo et al. 1997).

Other measurements of the water production rate come from observations of its photodissociation products. The $A^{2} \Sigma^{+}-X^{2} \Pi_{i}(0,0)$ band of $\mathrm{OH}$ near 3090 $\AA$ observed with the International Ultraviolet Explorer (IUE) gives $Q_{\mathrm{H}_{2} \mathrm{O}}=1.6-1.9 \times 10^{29} \mathrm{~s}^{-1}$ (Festou et al. 1997) for the March 23.1-25.1 period. The same band monitored from the ground by narrowband photometry (Schleicher 1997) yields $Q_{\mathrm{H}_{2} \mathrm{O}}=$ 2.5, 2.7 and $1.7 \times 10^{29} \mathrm{~s}^{-1}$ on March 23.5, 24.5 and 25.1 UT respectively, using model parameters pertaining to the $\mathrm{OH}$ collisional coma sampled by the instrumental aperture (Table 5). $\mathrm{OH}$ was also observed in the radio from its maser lines at 1667 and $1665 \mathrm{MHz}$ (Gérard et al. 1998), from which $Q_{\mathrm{H}_{2} \mathrm{O}}$ was estimated to be $\sim 2 \times 10^{29} \mathrm{~s}^{-1}$ on March 24.1, and $\sim 1.4 \times 10^{29} \mathrm{~s}^{-1}$ on March 26.1. From observation 
of HI Lyman- $\alpha$, emission with the SWAN instrument on board SOHO, Bertaux et al. (1998) derived $Q_{\mathrm{H}_{2} \mathrm{O}}$ $\sim 1.9 \times 10^{29} \mathrm{~s}^{-1}$. Finally, Hicks and Fink (1997) reported $\sim 3 \times 10^{29} \mathrm{~s}^{-1}$ from observations of the forbidden line of oxygen $\mathrm{OI}\left({ }^{1} D\right)$ at $6300 \AA$. However, this determination is hampered by the uncertainty in the branching ratio to $\mathrm{OI}\left({ }^{1} D\right)$ following $\mathrm{H}_{2} \mathrm{O}$ dissociation: it will not be considered here.

In summary, the water production rate, as estimated by various techniques, is between 1.6 and 2.7 $\times 10^{29} \mathrm{~s}^{-1}$ during the period of our HDO observations. Many of these results are of preliminary nature and further work may lead to a better agreement between the different estimates. It is presently difficult to make a choice between the various measurements, because each value relies on state-of-the-art models and assumptions. Even the determination made from the direct observation of water by its $\nu_{1}+\nu_{2}+\nu_{3}-\nu_{1}$ band should be taken with a grain of salt, because the strength of this band is unknown and the results rely on the assumption that it is identical to that of the $\nu_{2}+\nu_{3}$ band.

The various data sets show significant temporal variations in the water production rate during the March 20 to 25 period (reaching $50 \%$ or more), which may be linked to the disruption of the nucleus observed in the visible (Jorda et al. 1996). Most agree with a surge of outgassing associated with the fragmentation, followed by a plateau or a decrease in activity (Bertaux et al. 1998; Gérard et al. 1998; Schleicher 1997; Table 5). Such temporal variations were also seen in the production rates of other species, such as HCN (Lis et al. 1997). In addition, a periodic modulation of $\sim 20 \%$ amplitude, related to the 6.2 hours nucleus rotation, is observed in the $\mathrm{OH}$ gas production curve monitored by narrow-band photometry (Schleicher 1997; Schleicher et al. 1998). The calculation of the $Q_{\mathrm{HDO}} / Q_{\mathrm{H}_{2} \mathrm{O}}$ ratio thus requires a comparison of the amounts of $\mathrm{HDO}$ and $\mathrm{H}_{2} \mathrm{O}$ emitted at the same time. The comparison between various data sets is not easy due to the difference in the fields of view (from a few arcsec to several degrees) and the time lag between production of $\mathrm{HDO}$ and $\mathrm{H}_{2} \mathrm{O}$ and of the water photodissociation products. We note that the decrease seen in the HDO line intensity between March 23.5 and 24.5 (Table 3), which is marginal taking into account combined uncertainties, is neither observed in the $\mathrm{OH}$ data of Schleicher (1997) obtained with a similar field of view, nor in the $\mathrm{CH}_{3} \mathrm{OH}$ lines observed in the HDO spectrum (Lis al. 1997).
Using a water production rate of $(2.1 \pm 0.5) \times 10^{29}$ $\mathrm{s}^{-1}$ (average of the values given above) together with the average HDO production rate for March 23-24 of $(1.20 \pm 0.28) \times 10^{26} \mathrm{~s}^{-1}$, we infer a $Q_{\mathrm{HDO}} / Q_{\mathrm{H}_{2} \mathrm{O}}$ ratio of $(57 \pm 19) \times 10^{-5}$, which corresponds to a $(D / H)_{\mathrm{H}_{2} \mathrm{O}}$ ratio of $(29 \pm 10) \times 10^{-5}$.

\subsection{Deuterium abundance in hydrogen cya- nide}

The $3 \sigma$ upper limit for the DCN $J(5-4)$ line intensity on March 26 (Table 1) corresponds to $Q_{\mathrm{DCN}}<$ $2.6 \times 10^{24} \mathrm{~s}^{-1}$. On the same date, the $J(4-3)$ line of HCN at $354.505 \mathrm{GHz}$ was observed, from which $Q_{\mathrm{HCN}}$ $=2.1-2.4 \times 10^{26} \mathrm{~s}^{-1}$ was derived (Lis et al. 1997). Both production rate determinations were made consistently, using the same model (Crovisier 1987) and model parameters. The upper limit for the $(D / H)_{\mathrm{HCN}}$ ratio in Hyakutake is thus approximately $1 \%$.

\section{Discussion}

The $\mathrm{D} / \mathrm{H}$ ratio measured in water in comet Hyakutake is consistent with the determination of $\mathrm{D} / \mathrm{H}$ in water in comet P/Halley obtained by NMS (Eberhardt et al. 1995) and IMS (Balsiger et al. 1995) aboard Giotto. It is also in agreement with the anal$y$ sis of the submillimeter detection of HDO in comet $\mathrm{C} / 1995 \mathrm{O} 1$ (Hale-Bopp) which gives $(33 \pm 8) \times 10^{-5}$ (Meier et al. 1998a). Note that, on the basis of their orbital elements, all three of these comets are believed to originate from the Oort cloud.

\subsection{Comparison with the interstellar medium}

The $\mathrm{D} / \mathrm{H}$ ratio in water in these comets appears to be enriched by a factor of at least 10 with respect to the protosolar value (Fig. 3 and 4). Geiss and Reeves (1981) pointed out long ago that high isotopic enrichments could not have occurred via reactions between neutral molecules in the primitive Solar Nebula; they showed that if a high enrichment of $\mathrm{D} / \mathrm{H}$ were discovered in solid objects in the Solar System, it would imply that the enrichment took place in the interstellar medium (ISM) through ion-molecule reactions, prior to the collapse of the protosolar cloud. More recently, it has been shown that high enrichments could also result from grain-surface processes in the ISM (Tielens 1983; Brown and Millar 1989).

Owen et al. (1986) were the first to present $\mathrm{D} / \mathrm{H}$ measurements demonstrating that highly enriched components are present in the outer Solar System. 


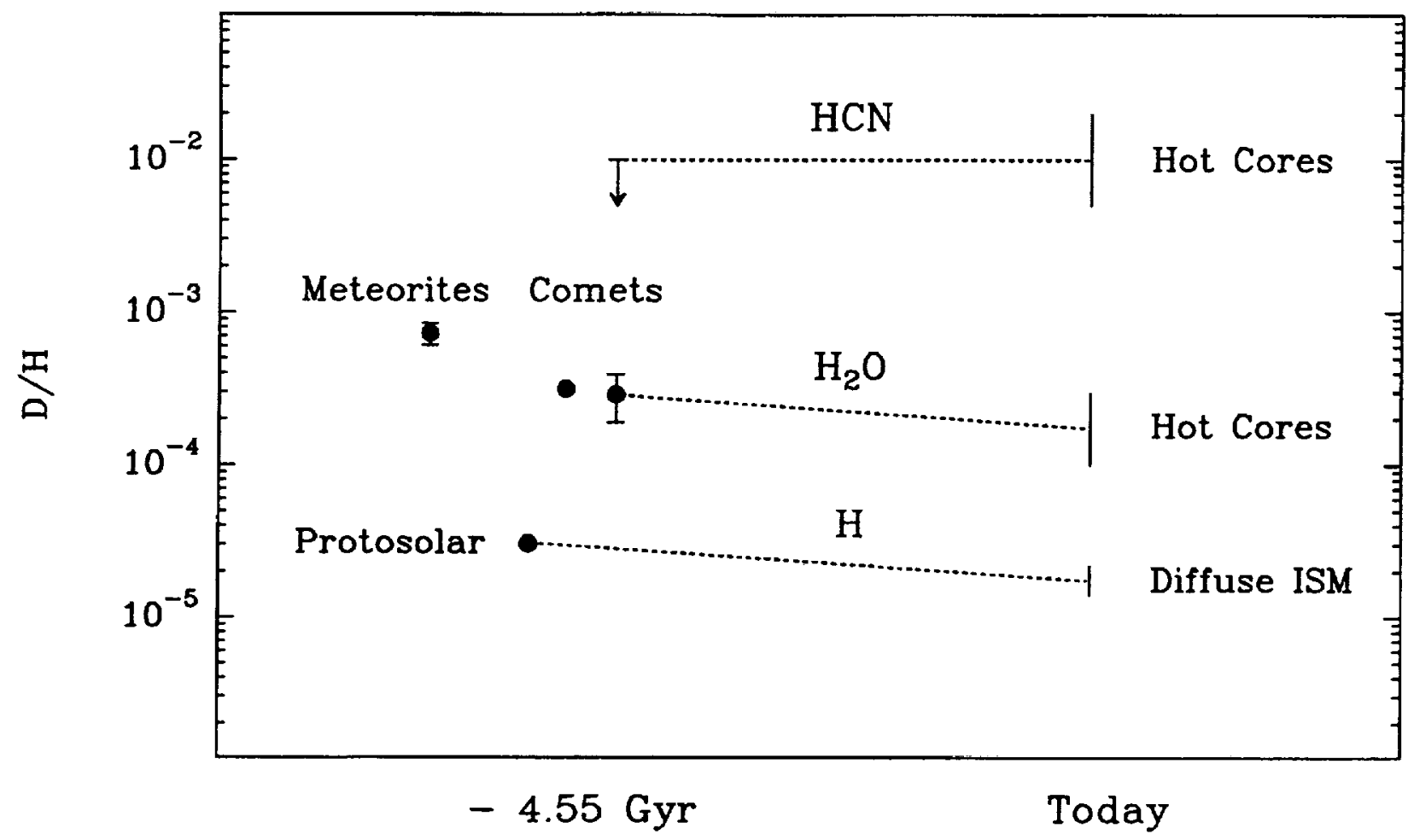

Fig. 3.- $\mathrm{D} / \mathrm{H}$ in protosolar cloud and ISM today. Only the high D-enrichment observed in meteorites is drawn. The $\mathrm{D} / \mathrm{H}$ ratio in cometary $\mathrm{H}_{2} \mathrm{O}$ measured in $\mathrm{P} / \mathrm{Halley}$ (Eberhardt et al. 1995; Balsiger et al. 1995) is the value given without an error bar; that measured in C/1996 B2 (Hyakutake) is the value given with an error bar. The vertical arrow is the upper limit on the $\mathrm{DCN} / \mathrm{HCN}$ ratio in comet Hyakutake. The sources of the other data are given in Section 4.

Although subsequent observations have led to changes in the derived values (Section 4.2), the basic point remains: if condensed matter has never been able to equilibrate with hydrogen gas through thermal reactions and mixing in the Solar Nebula, it will retain the values of $\mathrm{D} / \mathrm{H}$ set in the ISM. These values should differ from one species to another, reflecting the different binding energies of the deuterated molecules. Figure 3 compares cometary $\mathrm{D} / \mathrm{H}$ ratios with those observed in various components of the ISM. Note that values of interstellar D/H's represent the isotopic ratios in specific regions at the present stage of galactic evolution, whereas the cometary $\mathrm{D} / \mathrm{H}$ pertains to that of the protosolar cloud $4.55 \mathrm{Gyr}$ ago.

The $\mathrm{D} / \mathrm{H}$ ratio in hydrogen in the local ISM has been investigated through observations of the $\mathrm{Ly} \alpha$ lines of hydrogen and deuterium atoms in absorption toward several stars (Linsky et al. 1995; Wood et al. 1996; Piskunov et al. 1997). The average $\mathrm{D} / \mathrm{H}$ ratio is $\sim 1.6 \times 10^{-5}$ with the individual values ranging from 1.4 to $2.2 \times 10^{-5}$ (Piskunov et al. 1997). This is about a factor of 2 lower than the protosolar value, which reflects the local ISM deuterium abundance at the time and place of Solar System formation.

HDO has been observed in several galactic hot cores (Jacq et al. 1990; Helmich et al. 1996; Gensheimer et al. 1996). Hot core regions are parts of giant molecular clouds where high-mass stars are forming. In such warm regions, some species observed in the gas phase are believed to be produced by the sublimation of icy grain mantles (e.g. $\mathrm{H}_{2} \mathrm{O}, \mathrm{CH}_{3} \mathrm{OH}$ ), so that the gas composition of these species potentially traces the ice composition. HDO is observed through its $1_{01}-0_{00}$ submillimeter line as well as several millimeter lines. The abundance of $\mathrm{H}_{2} \mathrm{O}$ is measured through the $3_{13}-2_{20}$ line of $\mathrm{H}_{2}^{18} \mathrm{O}$ at $203 \mathrm{GHz}$. Most of the observed values correspond to $(\mathrm{D} / \mathrm{H})_{\mathrm{H}_{2} \mathrm{O}}$ in the range $10-30 \times 10^{-5}$, comparable to the observed $\mathrm{D} / \mathrm{H}$ 
ratio in cometary water.

DCN has also been observed in hot cores, as well as in cold galactic clouds with no or minimal star formation. The deuterium enrichment in $\mathrm{HCN}$ is found to be even higher than in $\mathrm{H}_{2} \mathrm{O}$ with $\mathrm{D} / \mathrm{H}$ close to 5-8 $\times 10^{-3}$ in hot cores, but as high as $2 \times 10^{-2}$ in cooler clouds as TMC1 or the cores in $\rho$ Ophiuchi (Wootten 1987; Mangum et al. 1991; Helmich et al. 1996). Although the hot core values are just below the upper limit we measured for the $\mathrm{DCN} / \mathrm{HCN}$ ratio in comet Hyakutake, our limit lies below the ratio observed in the coldest interstellar medium clouds. From the recent detection of DCN in C/1995 O1 (Hale-Bopp), cometary $(\mathrm{D} / \mathrm{H})_{\mathrm{HCN}}$ is $(2.3 \pm 0.4) \times 10^{-3}$ (Meier et al. 1998 b), i.e. significantly below the values observed in hot cores and cold clouds, although well above the protosolar $\mathrm{D} / \mathrm{H}$ value.

The chemistry necessary to produce such high levels of deuteration in the interstellar medium requires very low temperatures. The most current explanation given for the high D-enrichments observed in the warm (>100 K) environment of hot cores is that D-rich molecules formed and froze onto grains in an earlier low-temperature phase. Reactions with atomic deuterium on grain surfaces may also contribute (Tielens 1983). Another more recent suggestion is that water ice mantles could be created in interstellar clouds by the passage of a $\mathrm{C}$-type shock, followed by rapid hydrogenation of abundant gas-phase oxygen, and subsequent depletion of water onto the cold dust grains (Bergin et al. 1998). This model does offer an alternative method to create water-rich mantles. However, the level of deuterium fractionation is difficult to assess as a shock may alter the $\mathrm{HDO} / \mathrm{H}_{2} \mathrm{O}$ ratio, as will any post-shock evolution prior to depletion onto the grain surface.

In the hypothesis that grains infalling from the protosolar cloud onto the discoid nebula were never subsequently reprocessed or mixed with other components of the Solar Nebula (see discussion Sect. 4.3), the $\mathrm{D} / \mathrm{H}$ ratios observed in comets could have interesting implications. From models of deuterium chemistry in dense interstellar clouds (Millar et al. 1989), the lack of a highly deuterium-enriched component in cometary HCN would suggest that the outer parts of the protosolar cloud were warmer than $\sim 30 \mathrm{~K}$ when the gases condensed onto grains. These models give $(D / H)_{\mathrm{H}_{2} \mathrm{O}}$ ratios consistent with those observed in comets for temperatures of $\sim 50 \mathrm{~K}$. However, as discussed in Section 4.3, there are strong presump- tions that the $\mathrm{D} / \mathrm{H}$ ratios observed in comets are not unaltered relics of the protosolar chemistry.

\subsection{Comparison with other Solar System ob- jects}

A comparison of the deuterium abundance in comets with that in other Solar System bodies may have important cosmogonical implications. The $\mathrm{D} / \mathrm{H}$ ratio in objects relevant to our problem, namely in objects containing relics of enrichments which occurred in the nebula at the birth of the Solar System, are shown in Fig. 4, significantly updated from Lecluse et al. (1996). We do not include Mars and Venus which exhibit evidence of a strong atmospheric fractionation during the history of the planets, nor Titan since it was formed in the Saturn subnebula where chemical conversions and reprocessing of deuterium in carbon compounds presumably occurred (Prinn and Fegley 1981; Lunine and Tittemore 1993). New data shown in this figure concern the protosolar value, meteoritic data, comet Hyakutake, and preliminary results for Jupiter and Saturn from the Infrared Space Observatory (ISO).

The protosolar $\mathrm{D} / \mathrm{H}$ ratio has been estimated by several authors using the conversion of $\mathrm{D}$ to ${ }^{3} \mathrm{He}$ in the early Sun to trace the original D from measurements of the ${ }^{3} \mathrm{He} /{ }^{4} \mathrm{He}$ ratio in the solar wind (Geiss and Reeves 1972; Geiss 1993; Niemann et al. 1996). Gautier and Morel (1997) have recalculated the protosolar $\mathrm{D} / \mathrm{H}$ ratio by the same approach, but using a solar evolutionary model with microscopic diffusion. They derive $(\mathrm{D} / \mathrm{H})_{\text {proto }}=(3.01 \pm 0.17) \times 10^{-5}$. Geiss and Gloecker (1997) estimate that the helium isotopic fractionation results in lowering the ${ }^{3} \mathrm{He} /{ }^{4} \mathrm{He}$ ratio at the surface with respect to the solar wind value by $1-9 \%$, which leads to a lower limit for $(\mathrm{D} / \mathrm{H})_{\text {proto }}$ of approximately $2.6 \times 10^{-5}$. Accordingly, we conservatively assume $(\mathrm{D} / \mathrm{H})_{\text {proto }}=\left(3.0_{-0.4}^{+0.2}\right) \times 10^{-5}$.

The terrestrial $\mathrm{D} / \mathrm{H}$ reference value is currently $15.6 \times 10^{-5}$, measured in ocean water (SMOW) (De Witt et al. 1980). However, the $\mathrm{D} / \mathrm{H}$ ratio in oceans is expected to vary with time. Therefore, we prefer to adopt as a reference the averaged terrestrial value recently inferred by Lécuyer et $a l$. (1997) as equal to $(14.9 \pm 0.3) \times 10^{-5}$.

Recent analysis of meteoritic data reveals the presence of two different $D$-enrichments in water in the Semarkona and Bishunpur meteorites (Deloule et al. 1997, 1998). These authors find evidence of a minor 


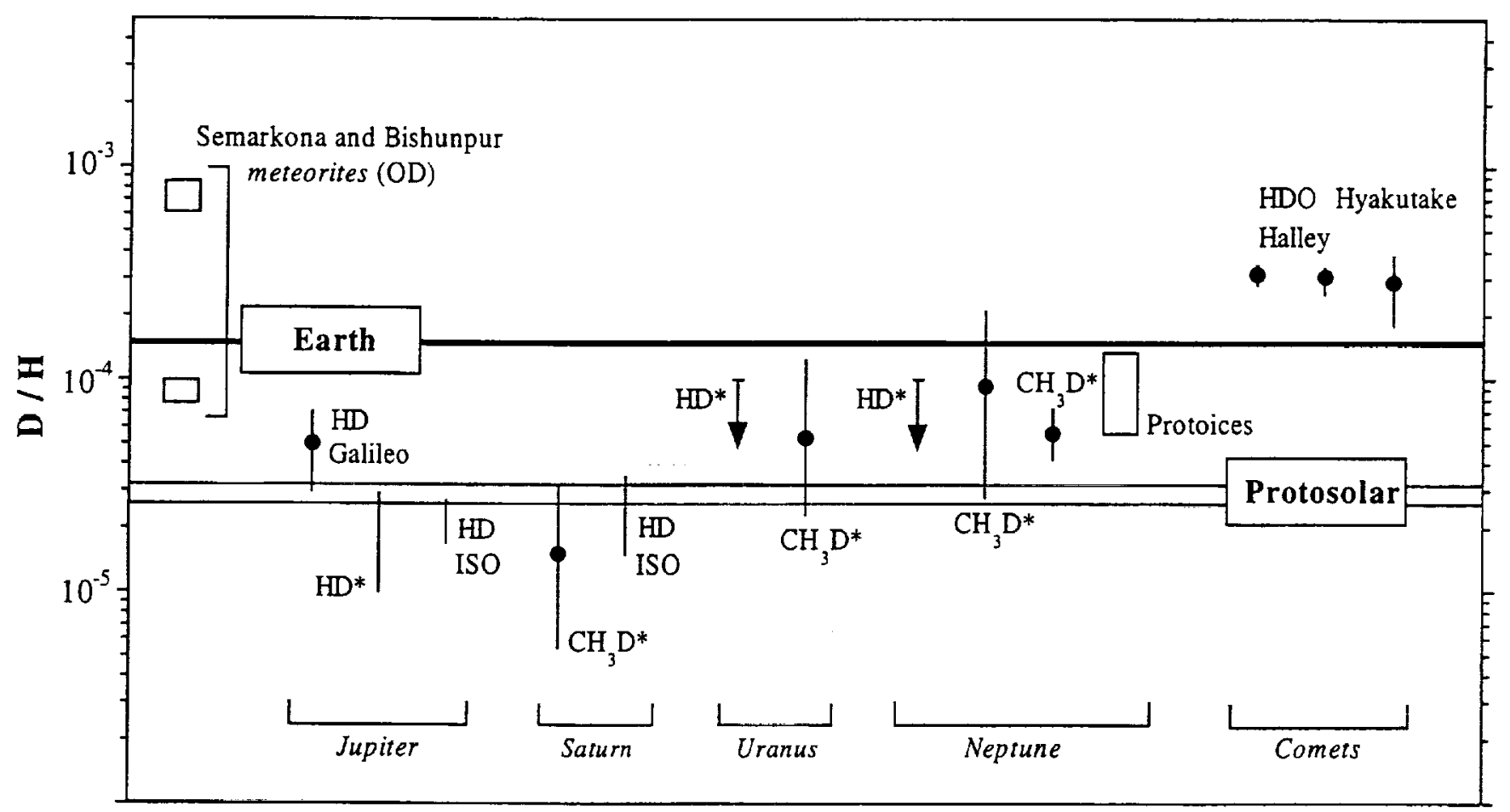

Fig. 4.- D/H ratios in the Solar System. The Earth value (Lécuyer et al. 1997) and the protosolar value (see Section 4.2) are shown for comparison. Asterisks indicate ground based observations. HD* for Jupiter is from Smith et al. (1989b). The vertical arrows labelled $\mathrm{HD}^{*}$ for Uranus and Neptune indicate upper limits derived by Smith et al. (1989a) from searches of HD lines from the ground. The values deduced from HD observations with ISO are from Encrenaz et al. (1996) (Jupiter) and Griffin et al. (1996) (Saturn); the value obtained by Galileo in Jupiter is from Niemann et al. (1996). The D/H ratio in methane given for Saturn and Uranus are from Noll and Larson (1990) and from de Bergh et al. (1986), respectively. Two values deduced from the measured $\mathrm{CH}_{3} \mathrm{D} / \mathrm{CH}_{4}$ ratio are shown for Neptune, that derived from de Bergh et al. (1990) from near-IR observations (large error bar) and that of Orton et al. (1992) (small error bar). The D/H ratio deduced for protoneptunian ices (see text) is drawn as a rectangular box. The measurements in the Semarkona and Bishunpur meteorites, which show the presence of two different D/H enrichments, are from Deloule et al. $(1997,1998)$. For comet $\mathrm{P} /$ Halley, are shown the values of Eberhardt et al. (1995) and Balsiger et al. (1995). The value given for C/1996 B2 (Hyakutake) is from this work.

(15\%) highly enriched component with $\mathrm{D} / \mathrm{H}=(73 \pm$ 12) $\times 10^{-5}$ and a major (85\%) moderately enriched component with $\mathrm{D} / \mathrm{H}=(8.8 \pm 1.1) \times 10^{-5}$, namely less than the Earth value of $14.9 \times 10^{-5}$.

As initially recognized by Hubbard and McFarlane (1980), the observed $\mathrm{D} / \mathrm{H}$ ratio in giant planets results from mixing of hydrogen originating from the primitive Solar Nebula (and thus having a protosolar $\mathrm{D} / \mathrm{H}$ ratio) with ices that may have been enriched in deuterium (for instance in the ISM) with respect to the protosolar value. In Jupiter and Saturn, the ice enrichment is currently considered negligible. However, Guillot et al. (1997) estimate that the amount of ices in Jupiter could be as high as 32 Earth masses 
compared to 318 Earth masses for the whole Jupiter mass. In such a case, a detectable enrichment cannot be ruled out. For instance a $\mathrm{D} / \mathrm{H}$ enrichment in proto-ices by a factor of 10 would result in an enrichment in Jupiter by $20 \%$ with respect to the protosolar value (Gautier and Morel 1997).

Preliminary results from ISO based on the detection of HD rotational lines on Jupiter and Saturn (Encrenaz et al. 1996; Griffin et al. 1996) are consistent with the protosolar value given above (Fig. 4). The preliminary results obtained by the mass spectrometer aboard the Galileo probe into Jupiter (Niemann et al. 1996) give a lower limit also consistent with the protosolar value. The accuracy of this measurement is expected to be improved when the spectrometer will have been fully calibrated. At this point, we do not know whether the deuterium abundance in Jupiter is representative of the protosolar value or not. Note that the various estimates of the $\mathrm{D} / \mathrm{H}$ ratio in Jupiter based on observations of $\mathrm{CH}_{4}$ are in conflict each other and spread out from $1 \times 10^{-5}$ (Gautier and Owen 1989) up to $5.5 \times 10^{-5}$ (Carlson et al. 1993; see Lecluse et al. 1996 for a detailed discussion and other references). For clarity, these are not included in Fig. 4.

The mass of hydrogen in Uranus and Neptune is much smaller than the mass of ices. If these planets were well mixed at least once in their history, as assumed in evolutionary models of Pollack and Bodenheimer (1989) or in the most recent models of their formation (Lissauer et al. 1995), water and hydrogen should be equilibrated at the high temperatures present in the interiors. In other words, the present hydrogen has acquired the deuterium enrichment initially present in protoices embedded in the nebula and which formed the cores of Uranus and Neptune. The $\mathrm{D} / \mathrm{H}$ ratio in protoices can be estimated (Lecluse et al. 1996) from models of planetary interiors which give the relative proportions of ices, rocks and gas. Following Eq. (34) of Lecluse et al. (1996), adopting the model of Podolak et al. (1995) for the interior of Neptune, and taking the most precise determination of $\mathrm{D} / \mathrm{H}$ in the atmosphere, namely the value of Orton et al. (1992) from $\mathrm{CH}_{3} \mathrm{D}$, and $(\mathrm{D} / \mathrm{H})_{\text {proto }}=$ $\left(3.0_{-0.4}^{+0.2}\right) \times 10^{-5}$, we obtain a $\mathrm{D} / \mathrm{H}$ ratio in protoneptunian ices of $\left(9.0_{-3.4}^{+4.5}\right) \times 10^{-5}$. This value is comparable with the moderately enriched component in the Semarkona and Bishunpur meteorites. Note that the recent measurements of the HD line at $37.7 \mu \mathrm{m}$ in Neptune from ISO (Feuchtgruber et al. 1997) is in good agreement with the Orton et al. value. The present estimate of $\mathrm{D} / \mathrm{H}$ in Uranus is uncertain, but an upper limit of $10 \times 10^{-5}$ derived from observations of HD lines in the visible suggests that proto-uranian ices were significantly less enriched in deuterium than cometary ices.

In summary, two categories of fossil deuterium enrichment in water appear in the Solar System: (i) a moderate deuterium enrichment (a factor of $\sim 3$ with respect to the protosolar value) is found in protoneptunian ices and the low enriched component of Semarkona and Bishunpur; (ii) a high deuterium enrichment is found in comets (a factor of $\sim 10$ ), and the highly enriched component of Semarkona and Bishunpur (a factor of $\sim 25$ ).

\subsection{Implications for the origin of comets}

As discussed previously, the high deuterium enrichment in water can be obtained only through ionmolecule or grain-surface reactions occurring in the interstellar medium. However, an enrichment up to a factor of 3 can also be reached in the Solar Nebula (when neglecting diffusion) through thermal isotopic exchange (Lecluse and Robert 1994). Detailed calculations based on the generalization of the model of Dubrulle (1993) are out of the scope of this report and will be presented in a forthcoming paper (Drouart et al. 1998). It is assumed in this latter work that the transport of small icy particles and water vapor in the turbulent nebula is diffusive and that small particles remain mixed with the gas (Dubrulle et al. 1995). It is also assumed that ices infalling from the protosolar cloud on the nebula disk are strongly enriched in deuterium, by a factor of about 25 as in the highly enriched component of the Semarkona and Bishunpur meteorites. Preliminary results indicate that the diffusion occurring in the nebula that is required to transport the angular momentum by turbulent viscosity to Neptune redistributes the deuterium enrichment uniformly out to the orbit of this planet. Accordingly, an enrichment of $\sim 3$ could have occurred at $3 \mathrm{AU}$ where presumably meteorites were formed, as well as at $30 \mathrm{AU}$. But it is not possible to obtain an enrichment by a factor of 3 in one part of a turbulent nebula and by a factor of 10 in another part of the same turbulent nebula, except very briefly in the outer part of the early nebula when the mixing was not completely achieved.

From these results, there are two possible scenarios, as detailed in Drouart et al. (1998): 
(i) Icy cometesimals might have been rapidly formed - in less than 1 or $2 \times 10^{5}$ years - in the Uranus-Neptune distance range, as assumed in standard models of cometary formation (Fernandez and Ip 1981; Duncan et al. 1987). At this time, the mixing is not yet complete and the deuterium enrichment not yet uniform throughout the nebula. Comets of kilometer sizes are formed in a few hundred thousand years (Weidenschilling 1997). They are rapidly expelled towards the Oort cloud by early giant planets. Uranus and Neptune continue to grow for several millions years (Pollack et al. 1996), by accretion of planetesimals formed later than cometesimals from microscopic grains that are mixed with the nebula gas and exhibit a deuterium enrichment of the order of 3 , as in the low enriched component of the Semarkona and Bishunpur meteorites. This scenario requires an appropriate nebula, an appropriate temporal sequence for the formation and the expulsion of comets and a continuous formation of planetesimals from microscopic grains during the major part of the history of Uranus and Neptune. On the other hand, it permits us to explain the presence of crystalline silicates detected in several comets (Hanner et al. 1994) and recently clearly confirmed by ISO observations of Hale-Bopp (Crovisier et al. 1997). Hanner (1997) indicates a proportion of $20 \%$ of crystalline silicates with respect to amorphous silicates in comet HaleBopp. Silicates are observed in amorphous form in the ISM. Forming crystalline silicates requires temperatures higher than $1300 \mathrm{~K}$, impossible to reach in the outer nebula. A partial diffusive transport of these species from the inner nebula out to the UranusNeptune region is a plausible solution of the problem.

(ii) The nebula might have been turbulent out to the region of formation of Neptune, somewhere between 25 and $30 \mathrm{AU}$, since several authors consider that this planet may have migrated outward by several AU during its formation process (Fernandez and Ip 1996; Malhotra 1998). Beyond Neptune, the turbulence may have vanished and a laminar regime became predominant, implying a low mixing with the turbulent part of the nebula. This scenario is consistent with observations of disks around young protostars which exhibit sizes generally larger (up to $1000 \mathrm{AU}$ ) than assumed in current models of turbulent nebula (Beckwith et al. 1993; Strom et al. 1993). Grains infalling from the protosolar cloud on this outer nebula may have sublimated and condensed again, as discussed by Lunine et al. (1991), but the local temper- ature was low enough (Dubrulle 1993) that no isotopic exchange with hydrogen could occur. Thus, even in this case, the ices preserved the deuterium enrichment acquired in the interstellar medium. In this scenario, comets P/Halley, Hyakutake and HaleBopp would have been formed in this part of the nebula, namely around the location of the present Kuiper Belt or beyond. Levison and Duncan (1997) pointed out that a substantial part of the objects originating from the Kuiper belt may have become part of the Oort cloud. Interpreting the presence of crystalline silicates in comets requires also some mixing of the laminar nebula with the turbulent one, as observed in laboratory experiments (Daviaud et al. 1992) but not easily simulated numerically.

Discriminating between these scenarios requires improvements in the theories of the origin of comets, as well as additional measurements of $(D / H)_{\mathrm{H}_{2} \mathrm{O}}$ in both long-period and short-period comets. A low $\mathrm{D} / \mathrm{H}$ ratio in Jupiter family comets originating from the Kuiper Belt would imply that the nebula was turbulent much farther from Neptune and would favor scenario (i). A D/H ratio of the order of or larger than that in P/Halley, Hyakutake and Hale-Bopp would favor scenario (ii) and a common origin of comets beyond Neptune.

Whatever the scenario of cometary formation, it is clear that cometary ices have an interstellar origin, as previously advocated by a number of authors (Greenberg 1982; Mumma et al. 1993; Irvine et al. 1996). However, both scenarios (i) and (ii) assume that interstellar grains were partly mixed during cometary formation with water reprocessed in the warm inner part of the turbulent nebula. This provides an interpretation of the presence of crystalline silicates in some comets and also explains why the $(D / H)_{\mathrm{H}_{2} \mathrm{O}}$ values observed in the three Oort cloud comets are somewhat less than in the highly enriched component of the Semarkona and Bishunpur meteorites.

\subsection{Origin of water on the Earth}

The comparison of the $\mathrm{D} / \mathrm{H}$ ratios in comets and in meteorites with that of the Earth provides clues for the origin of the oceans of our planet. The high cometary values make it difficult to defend a major cometary origin of terrestrial water. On the contrary, in whole rock carbonaceous meteorites and in chondrules, the mean value of $\mathrm{D} / \mathrm{H}$, namely the average of the high enriched component and of the low enriched component, is consistent, within uncertainties, 
with the Earth value (Deloule et al. 1997; Lécuyer et al. 1997). The current assumption that planetesimals which formed the Earth were of chondritic composition favors the scenario of the formation of oceans mainly by outgassing from the Earth's surface. Some water on the Earth could be inherited from a cometary bombardment subsequent to its formation (Owen and Bar-Nun 1995) but the deuterium abundances presently measured in comets suggest that this contribution should not exceed $10 \%$ (Deloule et al. 1997) to $30 \%$ (Owen 1997).

\section{Conclusion}

We have measured a $(D / H)_{\mathrm{H}_{2}} \mathrm{O}$ ratio of $(29 \pm 10)$ $\times 10^{-5}$ in the water of C/1996 B2 (Hyakutake). We may expect in the future to reduce the uncertainty of this measurement, with better constraints on the water production rate of Hyakutake at the time of the HDO observations.

This $\mathrm{D} / \mathrm{H}$ ratio is consistent with the value found in $\mathrm{P} /$ Halley and more recently in Hale-Bopp (Meier et al. 1998a). The high enrichment found in these objects with respect to the protosolar value implies that ices which formed comets were initially enriched in the protosolar cloud through ion-molecule isotopic exchanges or grain-surface reactions, prior to the collapse of the cloud.

However, we believe that some mixing with ices reprocessed in the turbulent part of the nebula subsequently occurred due to diffusive processes. Indeed, the $D / H$ enrichment found in these three comets is somewhat less than that found in some components of primitive meteorites. The presence of crystalline silicates in comets, which requires very high temperatures achievable only in the inner part of the early nebula, favors this assumption.

On the basis of the $\mathrm{D} / \mathrm{H}$ enrichments in water found in other objects of the solar system, we discuss two possible scenarios for the origin of comets which have strong consequences for the structure of the nebula. Following Drouart et al. (1998), in both scenarios it is assumed that the transport of the angular momentum from the center of the nebula out to Neptune or farther was made by turbulent viscosity. Due to diffusion, the radial distribution of the deuterium enrichment in water became rapidly uniform throughout the nebula out to Neptune at least.

In the first scenario, comets $\mathrm{P} /$ Halley, Hyakutake and Hale-Bopp were formed very early in the his- tory of the nebula and rapidly expelled to the Oort cloud by growing giant planets, then keeping a part of the deuterium enrichment acquired in the protosolar cloud. Uranus and Neptune grew slowly during all the lifetime of the nebula by accreting planetesimals continuously formed during the whole story of the nebula and exhibiting a low deuterium enrichment. In this scenario, comets coming from the Uranus-Neptune region populated the Oort cloud.

In the second scenario, comets formed in the nonturbulent part of the nebula, in this outer region beyond Neptune, which was only weakly mixed with the inner nebula. Icy grains kept most of their interstellar deuterium enrichment, whatever the time after the formation of the nebula. Comets were subsequently expelled by giant planets towards the Oort cloud. The deuterium enrichment in ices which formed Uranus and Neptune was low due to turbulent mixing. In this scenario, all comets in the Oort cloud originate from the Kuiper Belt or beyond.

For the moment, we cannot discriminate between these two scenarios, since the $\mathrm{D} / \mathrm{H}$ ratio in comets originating from the Kuiper Belt has not yet been measured. In addition, the three comets in which HDO has been observed may not be representative of the whole population of the Oort cloud. A low $\mathrm{D} / \mathrm{H}$ ratio in Jupiter family comets originating from the Kuiper Belt would imply that the nebula was turbulent much farther from Neptune and would favor the first scenario. A D/H ratio of the order of or larger than that in $\mathrm{P} / \mathrm{Halley}$, Hyakutake and HaleBopp would validate the second scenario and favors a common origin of comets beyond Neptune. The presence of crystalline silicates in comets is consistent with both scenarios, provided that the mixing with the inner part of the nebula was large enough.

We conclude that it is extremely important to measure $\mathrm{D} / \mathrm{H}$ in $\mathrm{H}_{2} \mathrm{O}$ in every comet of sufficient brightness. Periodic comets may require visits by spacecraft and we look forward to the ROSETTA mission as the first such encounter.

\section{Acknowledgments}

We are grateful to Don Yeomans for providing us ephemerides of C/1996 B2. We thank François Robert for enlightening discussions and for providing his preprints prior to publication, and Nicolas Biver for including collisions with electrons in the HDO model. We also thank Bérangère Dubrulle for extensive discussions on the structure of the Solar 
Nebula and Alessandro Morbidelli for valuable discussions on the origin of comets. We are grateful to J.-L. Bertaux, N. Dello Russo, M. Festou, U. Fink and D. Schleicher, for providing us their water production rates before publication. This research has been supported by NSF grant AST 93-13929 to the Caltech Submillimeter Observatory and by the Programme national de planétologie de l'Institut national des sciences de l'univers and the Centre national de la recherche scientifique (URA 1757 and 264). Research at the National Astronomy and Ionosphere Center is supported by the National Science Foundation under a cooperative agreement with Cornell University.

\section{APPENDIX}

\section{A. Excitation and intensity of the HDO rota- tional lines}

Local thermodynamical equilibrium (LTE) is only valid in the inner coma where collisional excitation prevails. In the outer coma, the HDO rotational levels reach the fluorescence equilibrium resulting from the balance between solar excitation of vibrational bands and vibrational and rotational spontaneous decay. Modeling the full evolution of the excitation conditions of HDO in the coma is necessary to investigate to which extent observations sample the LTE region and how the rotational populations are affected by radiative decay and IR radiative pumping. Due to its strong dipole moment $\left(\mu_{A}=0.657 \mathrm{D}\right.$ and $\mu_{B}=1.732$ D), HDO belongs to the category of molecules (which also includes $\mathrm{H}_{2} \mathrm{O}$ and $\mathrm{HCN}$ ) whose rotational populations relax rapidly toward fluorescence equilibrium where only the lower states are significantly populated.

The model computes the evolution of the rotational population distribution within the ground vibronic state as the HDO molecules expand in the coma, encountering varying excitation conditions. This model is similar to those developed for e.g. $\mathrm{H}_{2} \mathrm{O}$ (BockeléeMorvan 1987), $\mathrm{H}_{2} \mathrm{CO}$ (Bockelée-Morvan and Crovisier 1992) and linear molecules (Crovisier 1987). The rate equations include thermal excitation by collisions, absorption of the solar IR radiation in the vibrational bands and spontaneous decay. In addition to collisions with water, the model also considers collisions with electrons (see Biver 1997, Biver et al. 1997). The main parameters of the model are the kinetic temperature of the gas, $T_{k i n}$, the total crosssection for rotational de-excitation by collisions with
$\mathrm{H}_{2} \mathrm{O}, \sigma_{c}$, and the outflow velocity $v_{\text {exp }}$. The Haser model is used to relate the density of water in the coma to its production rate $Q_{\mathrm{H}_{2} \mathrm{O}}$. Details on the assumed electron density $n_{e}$ and temperature $T_{e}$ are given in Biver (1997) and Biver et al. (1997). In summary, the electron density as a function of distance to nucleus $r$ follows the behavior of the total ion density measured in $\mathrm{P} /$ Halley by ion mass spectrometers (Balsiger et al. 1986; Häberli et al. 1995) with (i) a recombination zone in the inner coma at $r<R_{C S}$ where the density varies according to $\left(Q_{\mathrm{H}_{2} \mathrm{O}} \sqrt{T_{e}}\right)^{1 / 2} / r$ and the electrons are thermalized to the temperature of the gas; (ii) a pile-up region beginning at $r=R_{C S}$ where the ions/electrons density increases as the result of increasing electron temperature and decreasing electronic recombination (Eberhardt and Krankowsky 1995); (iii) an external region at $r>R_{r e c}$ where the density follows the $Q / r^{2}$ dependence expected for radial expansion without significant additional ionization. $R_{C S}$ and $R_{\text {rec }}$ are scaled according to $Q_{\mathrm{H}_{2} \mathrm{O}}^{3 / 4}$ and $Q_{\mathrm{H}_{2} \mathrm{O}}^{1 / 2}$, respectively, and the absolute scaling is made such that the modeled $n_{e}$ reproduces the measurements in $\mathrm{P} /$ Halley for a water production rate of $6 \times 10^{29} \mathrm{~s}^{-1}$. With this model, $R_{C S}=3800 \mathrm{~km}$ and $R_{\text {rec }}=9000 \mathrm{~km}$ for comet Hyakutake at the time of the HDO observations.

Energy levels, rotational line frequencies and strengths of the ground vibrational state were taken from the JPL molecular data base (Pickett et al. 1996). For modeling radiative pumping of the vibrational bands, we used the spectroscopic data of the GEISA molecular data base (Husson et al. 1992). In the present work we only consider the three fundamental bands of vibration of HDO $\nu_{1}\left(2727 \mathrm{~cm}^{-1}\right), \nu_{2}$ $\left(1402 \mathrm{~cm}^{-1}\right)$ and $\nu_{3}\left(3707 \mathrm{~cm}^{-1}\right)$, whose total band strengths are 51, 209 and $129 \mathrm{~cm}^{-2}$ atm $^{-1}$, respectively. Harmonic and combination bands can be neglected on first order. This translates into solar excitation rates at $1 \mathrm{AU}$ from the Sun of $6.5 \times 10^{-5}$, $1.7 \times 10^{-4}$ and $1.9 \times 10^{-4} \mathrm{~s}^{-1}$, respectively. The code includes 80 rotational levels in the ground state $\left(E<943 \mathrm{~cm}^{-1}\right)$ and in each of the vibrational states.

Figure 5 shows how the fractional populations of the HDO rotational levels evolve from thermal to fluorescence equilibrium. Calculations were performed with model parameters pertaining to comet C/1996 B2 (Hyakutake) at it closest approach from the Earth $\left(r_{h}=1 \mathrm{AU}, Q_{\mathrm{H}_{2} \mathrm{O}}=2 \times 10^{29} \mathrm{~s}^{-1}\right)$. Collisions with electrons were not considered in this calculation and 


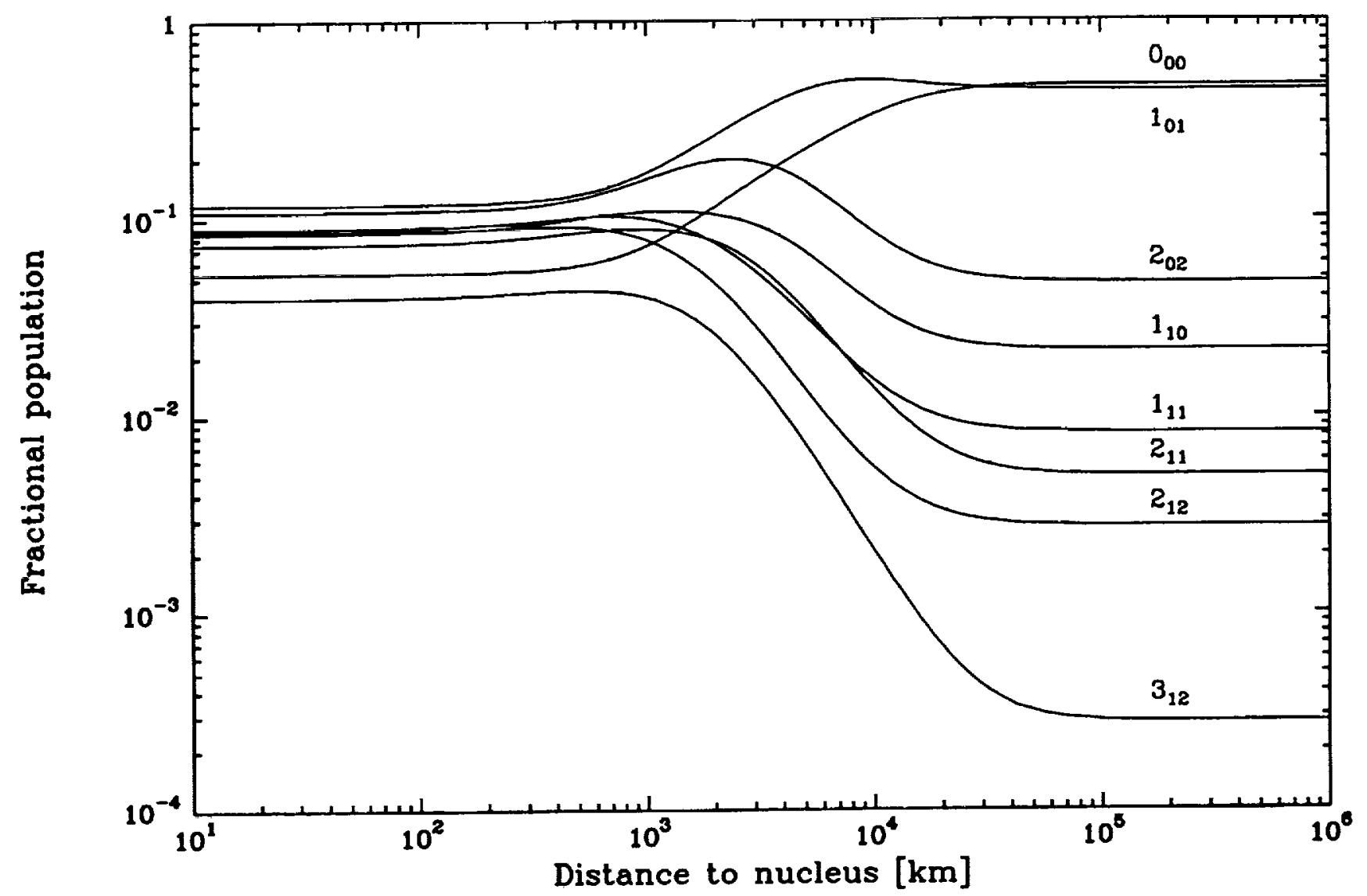

Fig. 5.- Evolution of the population of the rotational levels of $\mathrm{HDO}$ as a function of distance to nucleus. Calculations are performed with $Q_{H_{2} O}=2 \times 10^{29} \mathrm{~s}^{-1}, r_{h}=1 \mathrm{AU}, v_{e x p}=0.75 \mathrm{~km} \mathrm{~s}^{-1}, \sigma_{c}=5 \times 10^{-14} \mathrm{~cm}^{2}$ and $T_{k i n}$ $=70 \mathrm{~K}$. Collisions with electrons are not considered.

$\sigma_{c}$ was taken to be $5 \times 10^{-14} \mathrm{~cm}^{2}$. Thermal equilibrium breaks down at typically $500-1000 \mathrm{~km}$ from the nucleus for the low-lying rotational levels shown in the figure. This corresponds to the distance at which spontaneous emission rates begin to exceed collision rates. Radiative lifetimes are a few hundred seconds or less and the molecules relax rapidly to populate the lower states. At fluorescence equilibrium $\left(\tau_{h}=\right.$ $1 \mathrm{AU}$ ), only the $0_{00}$ and $1_{01}$ are significantly populated, with $90 \%$ of the total population distributed among these two levels. This is because at $r_{h}=1$ $\mathrm{AU}$, the IR excitation rate that pumps the $l_{01}$ level is comparable to its spontaneous emission rate $\left(A_{i j}\right.$ $\left.=1.747 \times 10^{-4} \mathrm{~s}^{-1}\right)$. Between two fluorescence cycles, the HDO molecule has time to cascade down to the $l_{01}$ rotational level, but not to fully relax down to the $0_{00}$ level. A fully relaxed population distribu- tion would be obtained at large heliocentric distances, while, well below $1 \mathrm{AU}$, a large number of levels would be significantly populated at fluorescence equilibrium.

In Table 6, we give predicted brightness temperatures for a selection of HDO millimeter and submillimeter transitions observable from ground-based telescopes. This may help for planning observations. We only consider two favorable cases for detection: 1) a moderately bright comet at $1 \mathrm{AU}$ from the Sun making a close approach to Earth (case of C/1996 $\mathrm{B} 2$ (Hyakutake)); 2) an active comet at $1 \mathrm{AU}$ from the Sun and from the Earth (case of $\mathrm{C} / 199501$ (Hale-Bopp)). Because in both cases the observations are sensitive to the intermediate region where neither LTE nor fluorescence equilibrium apply, we also give brightness temperatures calculated in these two limited assumptions. Calculations without elec- 
tronic collisions are also given for comparison.

\section{REFERENCES}

Balsiger, H., K. Altwegg, F. Buhler, J. Geiss, A.G. Ghielmetti, B.E. Goldstein, R. Goldstein, W.T. Huntress, W.-H. Ip, A. Lazarus, A. Meier, M. Neugebauer, U. Rettenmund, H. Rosenbauer, R. Schwenn, R.D. Sharp, E.G. Shelly, E. Ungstrup and D.T. Young 1986. Ion composition and dynamics at comet Halley. Nature 321, 330-334.

Balsiger, H., K. Altwegg, and J. Geiss 1995. $\mathrm{D} / \mathrm{H}$ and ${ }^{18} \mathrm{O} /{ }^{16} \mathrm{O}$ ratio in the hydronium ion and in neutral water from in situ ion measurements in comet P/Halley. J. Geophys. Res. 100, 5827-5834.

Beckwith S.V.W., and A.A.I. Sargent 1993. The occurrence and properties of disks around young stars. In Protostars and Planets III (E. H. Levy and J. I. Lunine, Eds.), pp. 521-541. Univ. of Arizona Press, Tucson.

Bergin, E.A., G.J. Melnick and D.A. Neufeld 1998. Post-shock chemical lifetimes of outflow tracers and a possible new mechanism to produce water ice mantles. Astrophys. J.. Submitted.

Bertaux, J.L., J. Costa, E. Quémerais, R. Lallement, E. Kyrola, W. Schmidt, T. Summanen, T. Makkinen, and C. Goukenleuque 1998. Lyman-alpha observations of comet Hyakutake with SWAN on SOHO. Planet. Space Sci.. In press.

Biver, N. 1997. Molécules Mères Cométaires: Observations et Modélisations. $P h D$ dissertation, Université Paris 7.

Biver, N., D. Bockelée-Morvan, P. Colom, J. Crovisier, D. Despois and G. Paubert 1997. Hydrogen cyanide in comets 23P/Brorsen-Metcalf, C/1989 X1 (Austin), C/1990 K1 (Levy) and 109P/ Swift-Tuttle. In preparation.

Bockelée-Morvan, D. 1987. A model for the excitation of water in comets. Astron. Astrophys. 181, 169-181.

Bockelée-Morvan, D., and J. Crovisier 1992. Formaldehyde in comets: II. Excitation of the rotational lines. Astron. Astrophys. 264, 282-291.

Bockelée-Morvan, D., J. Crovisier, P. Colom, and D. Despois 1994. The rotational lines of methanol in comets Austin 1990V and Levy 1990XX. Astron. Astrophys. 287, 647-665.

Brown P.D. and T.J. Millar 1989. Models of the gas-grain interaction - Deuterium chemistry. Mon. Not. R. Astro. Soc. 237, 661-671.

Cochran, A.L., and D.G. Schleicher 1993. Observational constraints on the lifetime of cometary $\mathrm{H}_{2}$ O. Icarus 105, 235-253.

Carlson, B.E, A.A. Lacis and W.B. Rossow 1993. Tropospheric gas composition and cloud structure of the Jovian north equatorial belt. J. Geophys. Res 98, 5251-5290.

Crovisier, J. 1987. Rotational and vibrational spectra of linear parent molecules in comets. Astron. Astrophys. Suppl. Ser. 68, 223-258.

Crovisier, J. 1994. Photodestruction rates for cometary parent molecules. J. Geophys. Res. Planets 99, E4, 3777-3781.

Crovisier, J., K. Leech, D. Bockelée-Morvan, T.Y. Brooke, M.S. Hanner, B. Altieri, H.U. Keller and E. Lellouch 1997. The spectrum of comet Hale-Bopp (C/1995 O1) observed with the Infrared Space Observatory at 2.9 astronomical units from the Sun. Science 275, 1904-1907.

Daviaud, F., J. Hegset and P. Berge 1992. Subcritical transition to turbulence in plane couette flow. Phys. Rev. Lett. 69, 2511-2514.

De Bergh, C., B.L. Lutz, T. Owen, J. Brault and J. Chauville 1986. Monodeuterated methane in the outer solar system. II. Its detection on Uranus at $1.6 \mu \mathrm{m}$. Astrophys. J. 311, 501-510.

De Bergh, C., B.L. Lutz, T. Owen and J.P. Maillard 1990. Monodeuterated methane in the outer solar system. IV. Its detection and abundance on Neptune. Astrophys. J. 355, 661-666.

Dello Russo, N., M.J. Mumma, M.A. DiSanti, K. Magee-Sauer, R. Novak, T. Rettig and M. Fomenkova 1997. Private communication.

Deloule, E., J.-P. Doukhan and F. Robert 1997. An interstellar isotopic signature recorded in altered pyroxene chondrules. 28th Lunar and Planet. Sci. Conf. 291-292.

Deloule E., J.-P. Doukhan and F. Robert 1998. Interstellar hydroxyl in meteoritic chondrules: implications for the origin of water in the inner solar system. Preprint. 
DeLucia, F.C., R.L. Cook, P. Helminger and W. Gordy 1971 . Millimeter and submillimeter wave rotational spectrum and centrifugal distortion effects of HDO. J. Chem. Phys. 55, 5334-5339.

De Witt, J.P., C.M. Van der Straaten and W.G. Mook 1980 . Determination of the absolute D/H ratio of V-SMOW and SLAP. Geostand. Newsletter 4, 33-36.

Drouart, A., B. Dubrulle, D. Gautier and F. Robert 1998. Structure and transport in the Solar Nebula from constraints on deuterium enrichment and giant planet formation. In preparation.

Dubrulle, B. 1993. Differential rotation as a source of angular momemtum transfer in the Solar Nebula. Icarus 106, 59-76.

Dubrulle, B., G. Morfill and M. Sterzik 1995. The dust subdisk in the protoplanetary nebula. Icarus 114, 237-246.

Duncan, M., T. Quinn and S. Tremaine 1987. The formation and extent of the Solar System comet cloud. Astron. J. 94, 1330-1338.

Eberhardt, P. and D. Krankowsky 1995. The electron temperature in the inner coma of $\mathrm{P} /$ Halley. Astron. Astrophys. 295, 795-807.

Eberhardt, P., U. Dolder, W. Schulte, D. Krankowsky, J.H. Lämmerzahl, J.H. Hoffmann, R. Hodges, J.J. Berthelier and J.M. Illiano 1987. The D/H ratio in water from Halley. Astron. Astrophys. 187, 435-437.

Eberhardt, P., M. Reber, D. Krankowsky and R.R. Hodges. 1995. The $\mathrm{D} / \mathrm{H}$ and ${ }^{18} \mathrm{O} /{ }^{16} \mathrm{O}$ ratios in water from comet $\mathrm{P} /$ Halley. Astron. Astrophys. 302, 301-316.

Encrenaz, Th, Th. de Graauw, S. Schaeidt, E. Lellouch, H. Feuchtgruber, D.A. Beintema, B. Bézard, P. Drossart, M. Griffin, A. Heras, M. Kessler, K. Leech, P. Morris, P.R. Roelfsema, M. Roos-Serote, A. Salama, V. Vandenbussche, E.A. Valentijn, G.R. Davis and D.A. Naylor 1996. First results of ISO-SWS observations of Jupiter. Astron. Astrophys. 315, L397-L400.

Fernandez, J.A. and W.-H Ip 1981. Dynamical evolution of a cometary Swarm in the outer planetary region. Icarus $47,470-479$.

Fernandez, J.A. and W.-H Ip 1996. Orbital expansion and resonant trapping during the accretion stages of the outer planets. Planet. Space Sci. 44, 431-439.

Festou, M.C., M.F. A'Hearn, H. Andernach, P.D. Feldman, R. Gonzales-Riestra, M. Haken, P. Rodriguez-Pascual, G.-P. Tozzi and H.A. Weaver 1997. In preparation.

Feuchtgruber, H., E. Lellouch, T. de Graauw, Th. Encrenaz and M. Griffin 1997. Detection of $\mathrm{HD}$ on Neptune and determinations of $\mathrm{D} / \mathrm{H}$ ratio from ISO/SWS observations. BAAS 29, 995.

Gautier, D. and P. Morel 1997. A Reestimate of the protosolar $\left({ }^{2} \mathrm{H} /{ }^{1} \mathrm{H}\right)_{p}$ ratio from $\left({ }^{3} \mathrm{He} /{ }^{4} \mathrm{He}\right)_{s W}$ solar wind measurements. Astron. Astrophys. 323, L9-L12.

Gautier, D. and T. Owen 1989. The composition of outer planets atmospheres. In Origin and Evolution of Planetary and Satellite Atmospheres (S.K. Atreya, J.B. Pollack and M.S. Matthews, Eds), pp. 487-512, Univ. of Arizona Press, Tucson.

Geiss, J. 1993. Primordial abundance of hydrogen and helium isotopes. In Origin and Evolution of the Elements (N. Pranzos, E. Vangioni-Flam and M. Casse, Eds), pp 89-106, Cambridge Univ. Press, Cambridge.

Geiss, J. and G. Gloecker 1997. Deuterium and Helium-3 in the protosolar cloud from solar wind measurements. In ISSI Workshop on Primordial Nuclei and Galactic Evolution, Bern (Switzerland), 6-10 May 1997.

Geiss, J. and H. Reeves 1972. Cosmic and Solar System abundances of deuterium and helium-3. Astron. Astrophys. 18, 126-132.

Geiss, J. and H. Reeves 1981. Deuterium in the Solar System. Astron. Astrophys. 93, 189-199.

Gensheimer, P.D., R. Mauersberger and T.L. Wilson 1996. Water in galactic hot cores. Astron. Astrophys. 314, 281-294.

Gérard, E., J. Crovisier, P. Colom, N. Biver, D. Bockelée-Morvan and H. Rauer 1998. Observations of the $\mathrm{OH}$ radical in comet $\mathrm{C} / 1996 \mathrm{~B} 2$ (Hyakutake) with the Nançay radio telescope. Planet. Space Sci.. In press.

Guillot, T., D. Gautier and W.B. Hubbard 1997. New constraints on the composition of Jupiter from Galileo measurements and interior models. Icarus 130, 534-539. 
Greenberg, J.M. 1982. What are comets made of? - A model based on interstellar dust. In Comets (L.L. Wilkening, Ed.), pp 131-163, Univ. of Arizona Press, Tucson.

Griffin, M. J., D.A. Naylor, G.R. Davis, P.A.R. Ade, P.G. Oldman, B.M. Swinyard, D. Gautier, E. Lellouch, G.S. Orton, Th. Encrenaz, T. de Graauw, I. Furniss, H. Smith, C. Armand, M. Burgdorf, A. Di Giorgio, D. Ewart, C. Gry, K.J. King, T. Lim, S. Molinari, M. Price, S. Sidher, A. Smith, D. Texier, N. Trams, S.J. Unger and A. Salama 1996. First detection of the $56 \mu \mathrm{m}$ rotational line of HD in Saturn's atmosphere. Astron. Astrophys. 315, L389-L392.

Häberli, Altwegg, K., H. Balsiger and J. Geiss 1995. Physics and chemistry of ions in the pile-up region of comet P/Halley. Astron. Astrophys. 297, 881-891.

Hanner, M.S. 1997. The dust properties in comet C/1885 O1 Hale-Bopp. BAAS 29, 1042.

Hanner, M.S., D.K. Lynch and R.W. Russel 1994. The 8-13 $\mu \mathrm{m}$ spectra of comets and the composition of silicate grains. Astrophys. J. 425, 274-285.

Helmich, E.P., E.F. van Dishoeck and D.J. Jansen 1996. The excitation and abundance of HDO towards $\mathrm{W} 3(\mathrm{OH}) /\left(\mathrm{H}_{2} \mathrm{O}\right)$. Astron. Astrophys. 313, 657-663.

Hicks, M.D. and U. Fink 1997. Spectrophotometry and the development of emissions for C/1996 B2 (comet Hyakutake). Icarus 127, 307-318.

Hubbard, W.B. and J.J. MacFarlane 1980. Theoretical predictions of deuterium abundances in the Jovian planets. Icarus 44, 676-681.

Husson, N., B. Bonnet, N.A. Scott and A. Chedin 1992. Management and Study of Spectroscopic Information - The GEISA program. J. Quant. Spectrosc. Rad. Transfer 48, 509-518.

Irvine, W., D. Bockelée-Morvan, D.C. Lis, H.E. Matthews, N. Biver, J. Crovisier, J.K. davies, W.R.F. Dent, D. Gautier, P.D. Godfrey, J. Keene, A.J. Lowell, T.C. Owen, T.G. Phillips, H. rauer, F.P. Schloerb, M. Senay and K. Young 1996. Spectroscopic evidence for interstellar ices in comet Hyakutake, Nature 382, 418-420.

Irvine, W. M. and R. F. Knacke 1989. The chemistry of interstellar gas and grains. In Origin and Evolution of Planetary and Satellite Atmospheres (S. K.
Atreya, J. B. Pollack and M. S. Matthews, Eds), pp. 3-34, Univ. of Arizona Press, Tucson.

Jacq, T., C.M. Walmsley, C. Henkel, A. Baudry, R. Mauersberger, and P.R. Jewell 1990. Deuterated water and ammonia in hot cores. Astron. Astrophys. 228, 447-470.

Jorda, L., J. Lecacheux, F. Colas, P. Laques, E. Frappa, A. Enzian and J. Klinger 1996. Coma structures of C/1996 B2 Hyakutake monitored at Pic du Midi. In Asteroids, Comets, Meteors 1996, Versailles 8-12 July 1996, COSPAR Colloquium 10.

Lecluse, C. and F. Robert 1994. Hydrogen isotope exchange reaction rates: origin of water in the Solar System. Geochim. Cosmochim. Acta 58, 2927-2939.

Lecluse, C., F. Robert, D. Gautier, and $M$. Guiraud 1996. Deuterium enrichment in giant planet. Planet. Space Sci., 44, 1579-1592.

Lécuyer, C., Ph. Gillet and F. Robert 1997. Hydrogen isotope composition of sea water and the global water cycle. Earth and Planet Sci. Lett.. In press.

Levison, H.L. and M.J. Duncan 1997. From the Kuiper belt to Jupiter family comets: the spatial distribution of ecliptics comets. Icarus 127, 13-32.

Linsky, J.L., A. Diplas, B.E. Wood, A. Brown, T.R. Ayres and B.D. Savage 1995. Deuterium and the local interstellar medium: properties for the Procyon and capella lines of sight. Astrophys. J. 451, 335-351.

Lis, D.C., J. Keene, T.G. Phillips, E. Bergin, P. Goldsmith, D. Bockelée-Morvan, J. Crovisier, D. Gautier, A. Wootten, D. Despois and T. Owen 1996a. C/1996 B2 Hyakutake. IAU Circular 6362 .

Lis, D.C., J. Keene, K. Young, T.G. Phillips, P.F. Goldsmith, E.A. Bergin D. BockeléeMorvan and J. Crovisier 1996b. Morphology, kinematics, and excitation of molecular gas in comet Hyakutake (C/1996 B2). In Asteroids, Comets and Meteors 1996, 8-12 July 1996, Versailles, France.

Lis, D.C., J. Keene, K. Young, T.G. Phillips, D. Bockelée-Morvan, J. Crovisier, P. Shilke, P.F. Goldsmith and E.A. Bergin 1997. Spectroscopic observations of comet C/1996 B2 (Hyakutake) with the Caltech Submillimeter Observatory. Icarus. $130,355-372$. 
Lissauer, J.J., J.B. Pollack, G.W. Wetherill and D.J. Stevenson 1995. Formation of the Neptune system. In Neptune and Triton (D.P. Cruikshank, Ed), pp. 37-108, Univ. of Arizona Press.

Lunine, J.I., S. Engel, B. Rizk and M. Horanyi 1991. Sublimation and reformation of icy grains in the primitive Solar Nebula. Icarus 94, 333-344.

Lunine, J.I. and W.C. Tittemore 1993. Origin of outer-planet satellites. In Protostars and Planets III (E.H. Levy and J.I. Levy, Eds), pp. 1149-1176, Univ. of Arizona Press.

Malhotra, R. 1998. Implications of the Kuiper Belt Structure for the Solar System. In Asteroids, Comets, Meteors 1996 (A.-C. Levasseur-Regourd , Ed), Pergamon-Elsevier), Cospar Colloquium 10, PergamonElsevier. In press.

Mangum, J.G., R.L. Plambeck and A. Wootten 1991. Fossil DCN in Orion-KL. Astrophys. J. 369, 169-174.

Marsden, B. 1996. C/1996 B2 Hyakutake. IAU Circular 6304.

Meier, R., T.C. Owen, D.C. Jewitt, H. Matthews, M. Senay, N. Biver, D. BockeléeMorvan, J. Crovisier and D. Gautier 1998b. Deuterium in Comet C/1995 O1 (Hale-Bopp): Detection of HCN. (Hale-Bopp). Preprint.

Meier, R., T.C. Owen, H.E. Matthews, D.C. Jewitt, D. Bockelée-Morvan, N. Biver, J. Crovisier and D. Gautier 1998a. A determination of the $\mathrm{HDO} / \mathrm{H}_{2} \mathrm{O}$ ratio in Comet $\mathrm{C} / 1995 \mathrm{O} 1$ (HaleBopp). Science 279, 842-844.

Millar, T.J., A. Bennett and E. Herbst 1989, Deuterium fractionation in dense interstellar clouds. Astrophys. J. 340, 906-920.

Mumma, M.J., M.A. DiSanti, N. Dello Russo, Fomenkova, M. Magee-Sauer K., C.D. Kaminski, and D.X. Xie 1996. Detection of abundant ethane and methane along with carbon-monoxide and water, in comet C/1996 B2 Hyakutake - Evidence for interstellar origin. Science 272, 1310-1314.

Mumma, M.J., P.R. Weissman and A.A. Stern 1993. Comets and the origin of the solar system Reading the Rosetta stone. In Protostars and Planets III (E.H. Levy and J.I. Levy, Eds), pp. 1177- 1252. Univ. of Arizona Press, Tucson.

Nakamura, T. and S. Nakano 1996. C/1996 B2 Hyakutake. IAU Circular 6299.
Niemann, H.B., H.B., S.K. Atreya, G.R. Carignan, T.M. Haberman, D.N. Harpold, R.E. Hartle, D.M. Huten, W.T. Kasprzak, P.R. Mahaffy, T.C. Owen, N.W. Spencer and S.H. Way 1996. The composition of Jupiter's atmosphere: a preliminary assessment by the Galileo probe mass spectrometer. Science 272, 846-848.

Noll, K.S. and P.L. Larson 1990. The spectrum of Saturn from 1990 to $2230 \mathrm{~cm}^{-1}$. Abundances of $\mathrm{AsH}_{3}, \mathrm{CH}_{3} \mathrm{D}, \mathrm{CO}, \mathrm{GeH}_{4}, \mathrm{NH}_{3}$ and $\mathrm{PH}_{3}$. Icarus 89, 168-189.

Orton, G.S., J.H. Lacy, J.M. Achterman, P. Parmar and W. S. Blass 1992. Thermal spectroscopy of Neptune: the stratospheric temperature, hydrocarbon abundances, and isotopic ratios. Icarus 100, 541-555.

Owen, T. 1997. From planetesimals to planets: contributions of icy planetesimals to planetary atmospheres. In From stardust to planetesimals (Y.J. Pendleton and A.G.G. Tielens, Eds), ASP Conferences Series 22, pp 435-450.

Owen, T. and A. Bar-Nun 1995. Comets, impacts and atmospheres. Icarus 116, 215-226.

Owen, T., B.L. Lutz and C. De Bergh 1986. Deuterium in the outer Solar System: evidence for two distinct reservoirs. Nature 320, 244-246.

Pickett, H.M., R.L. Poynter, E.A. Cohen, M.L. Delitsky, J.C. Pearson and H.S.P. Müller 1996. Submillimeter, Millimeter and Microwave Spectral Line Catalogue. JPL.

Piskunov, N., B.E. Wood, J.L. Linsky, R.C. Dempsey and T.R. Ayres 1997. Local interstellar properties and deuterium abundances for the lines of sight toward HR 1099, 31 Comae, $\beta$ Ceti, and $\beta$ Cassiopeia. Astrophys. J. 474, 315-328.

Podolak, M., A. Weizman and M. Marley 1995. Comparative models of Uranus and Neptune. Planet. Space Sci. 43, 1517-1522.

Pollack, J. B. and F. Bodenheimer 1989. Theories of the Origin and Evolution of Giant Planets. In Origin and Evolution of Planetary and Satellite Atmospheres (S. K. Atreya, J. B. Pollack, and M. S. Matthews, Eds.), pp. 565-602, Univ. of Arizona Press, Tucson.

Pollack, J.B., O. Hubicky, P. Bodenheimer, J.L. Lissauer, M. Podolak, and Y. Greenzweig 1996. Formation of the giant planets by concurrent accretion of solids and gas. Icarus 124, 62-85. 
Prinn, R.G. and B. Fegley 1981. Kinetic inhibition of $\mathrm{CO}$ and $\mathrm{N}_{2}$ reduction in circumplanetary nebulae: implications for satellite formation. Astrophys. J. 249, 308-317.

Schleicher, D.G. 1997. Private communication.

Schleicher, D.G., R.L. Millis, D.J. Osip, and S.M. Lederer 1998. Activity and the rotation period of comet Hyakutake (1996 B2). Icarus, in press.

Smith, M. D., W.V. Schempp, J. Simon, and K. H. Baines 1989a. The D/H ratio for Uranus and Neptune. Astrophys. J. 336, 962-966.

Smith, M. D., W.V. Schempp, and K. H. Baines 1989b. The D/H ratio for Jupiter. Astrophys. J. 336, 967-970.

Strom, S. E., S. Edwards and M. F. Skrutskie 1993. Evolutionary time scales for circumstellar disks associated with intermediate and solar-type stars. In Protostars and Planets III (E. H. Levy and J. I. Lunine, Eds.), pp. 837-866, Univ. of Arizona Press, Tucson.

Tielens, A.G.G.M. 1983. Surface chemistry of deuterated molecules. Astron. Astrophys. 119, 177184.

Watson, W.D. 1974. Ion-molecules reactions, molecule formation, and hydrogen isotopic exchange in dense interstellar clouds. Astrophys. J. 188, 35-42.

Weidenschilling, S.J. 1997. The origin of comets in the Solar Nebula: a unified model. Icarus 127, 290-306.

Wood, B.E., W.R. Alexander and J.L. Linsky 1996. The properties of the local interstellar medium and the interaction of the stellar winds of $\epsilon$ Indi and $\lambda$ Andromedae with the interstellar environment. Astrophys. J. 470, 1157-1171.

Wootten, A. 1987. Deuterated molecules in interstellar clouds. In Astrochemistry, IAU Symposium No. 120 (M.S. Vardya and S.P. Tarafdar, Eds.), pp. 311-319, Reidel, Dordrecht.

Xu, L.-H. and F.L. Lovas 1997. Microwave spectral tables. XXIV. Methanol $\left(\mathrm{CH}_{3} \mathrm{OH}\right.$ and $\left.{ }^{13} \mathrm{CH}_{3} \mathrm{OH}\right)$. J. Phys. Chem. Ref. Data 26, 17-140.

This 2-column preprint was prepared with the AAS IATEX macros $\mathrm{v} 4.0$. 
TABLE 1

LOG OF THE OBSERVATIONS.

\begin{tabular}{lccccc}
\hline \hline $\begin{array}{l}\text { Date } \\
{[\mathrm{UT}]}\end{array}$ & $\begin{array}{c}r_{h} \\
{[\mathrm{AU}]}\end{array}$ & $\begin{array}{c}\text { Frequency }^{\mathrm{b}} \\
{[\mathrm{AU}]}\end{array}$ & $\begin{array}{c}\text { Int. time } \\
{[\mathrm{min}]}\end{array}$ & $\begin{array}{c}T_{s y s} \\
{[\mathrm{GHz}]}\end{array}$ \\
\hline 960323.5 & 1.08 & 0.118 & 19 & 464.925 & 1500 \\
960324.5 & 1.06 & 0.105 & 31 & 464.925 & 1700 \\
960326.5 & 1.02 & 0.109 & 49 & 362.046 & 1200 \\
\hline
\end{tabular}

"Integration time on source.

${ }^{b}$ Central frequency of the main side band.

TABLE 2

Characteristics of the CSO telescope.

\begin{tabular}{ccc}
\hline $\begin{array}{c}\text { Frequency } \\
{[\mathrm{GHz}]}\end{array}$ & HPBW & $\eta_{m b}^{\mathrm{a}}$ \\
\hline 465 & 15. & 0.5 \\
362 & 20. & 0.7 \\
\hline
\end{tabular}

\&Half Power Beam Width.

bMain beam efficiency.

TABLE 3

LINE INTENSITIES

\begin{tabular}{|c|c|c|c|c|}
\hline Molecule & line & $\begin{array}{c}\text { Frequency } \\
\text { [GHz] }\end{array}$ & $\begin{array}{l}\text { date } \\
\text { [UT] }\end{array}$ & $\underset{\left[\mathrm{K} k \mathrm{~km} \mathrm{~s}^{-1}\right]}{\int T_{B} d v^{\mathrm{a}}}$ \\
\hline HDO & $1_{01}-0_{00}$ & 464.92452 & $\begin{array}{ccc}960323.5 \\
960324.5 \\
960323.5-24.5\end{array}$ & $\begin{array}{l}0.89 \pm 0.16 \\
0.47 \pm 0.15 \\
0.65 \pm 0.10\end{array}$ \\
\hline $\mathrm{DCN}$ & $5-4$ & 362.04648 & 960326.5 & $<0.18^{b}$ \\
\hline
\end{tabular}

a Main beam brightness temperature scale. The intensities and uncertainties are calculated on the high-resolution spectra over a 3 $\mathrm{km} \mathrm{s}^{-1}$ velocity interval.

$b_{3} \sigma$ upper limit. 
TABLE 4

COMA TEMPERATURE MEASUREMENTS

\begin{tabular}{|c|c|c|c|c|c|}
\hline Species & date & Lines & $\begin{array}{l}\text { FOV } \\
{[\mathrm{km}]}\end{array}$ & $\begin{array}{c}T_{\text {rot }} \\
{[\mathrm{K}]}\end{array}$ & Ref. $^{b}$ \\
\hline $\mathrm{CO}$ & March 24-25 & $346,461,691 \mathrm{GHz}$ & $800-1600$ & $73 \pm 6$ & (1) \\
\hline $\mathrm{CH}_{3} \mathrm{OH}$ & March 23-24 & $462,465 \mathrm{GHz}$ & 1200 & $69 \pm 10$ & (1) \\
\hline $\mathrm{CH}_{3} \mathrm{OH}$ & March 24 & $242 \mathrm{GHz}$ multiplet & 1600 & $75 \pm 7$ & (2) \\
\hline $\mathrm{CH}_{3} \mathrm{OH}$ & March 30 & $252 \mathrm{GHz}$ multiplet & 4200 & $65 \pm 7$ & (1) \\
\hline
\end{tabular}

a Rotational temperature.

b(1) From CSO observations, Lis et al. (1997); (2) from IRAM Plateau de Bure observations, Biver (1997).

TABLE 5

HDO, $\mathrm{H}_{2} \mathrm{O}$ PRODUCTION RATES AND $(\mathrm{D} / \mathrm{H})_{\mathrm{H}_{2} \mathrm{O}}$ RATIO

\begin{tabular}{|c|c|c|c|c|}
\hline Species & Date & {$\left[\begin{array}{c}Q \\
{\left[s^{-1}\right]}\end{array}\right.$} & Source & Reference \\
\hline $\mathrm{HDO}$ & March 23-24 & $(1.20 \pm 0.28) \times 10^{26}$ & Cso & this work \\
\hline $\mathrm{H}_{2} \mathrm{O}$ & $\begin{array}{c}\text { March 24.5 } \\
\text { March 25.1, 26.1 } \\
\text { March 23.1, 25.1 } \\
\text { March 23.5, 24.5, 25.2 } \\
\text { March 23, 26 } \\
\text { March 23, 24 }\end{array}$ & $\begin{array}{c}(2.3 \pm 0.3) \times 10^{29} \\
(2.0,1.4) \times 10^{29} \\
(1.6,1.9) \times 10^{29} \\
(1.6-2.5,1.7-2.7,1.0-1.7) \times 10^{29 b} \\
(1.8,1.9) \times 10^{29} \\
(3.1,2.8) \times 10^{29}\end{array}$ & $\begin{array}{c}\text { IRTF } / \mathrm{H}_{2} \mathrm{O} \\
\text { Nançay } / \mathrm{OH}^{\mathrm{a}} \\
\text { IUE/OH } \\
\text { Lowell Obs./OH } \\
\text { H Ly- } \alpha \\
\text { OI }\left({ }^{1} D\right)\end{array}$ & $\begin{array}{c}\text { Dello Russo et al. (1997) } \\
\text { Gérard et al. (1998) } \\
\text { Festou et al. (1997) } \\
\text { Schleicher (1997) } \\
\text { Bertaux et al. (1998) } \\
\text { Hicks and Fink (1997) }\end{array}$ \\
\hline$(\mathrm{D} / \mathrm{H})_{\mathrm{H}_{2}} \mathrm{O}$ & March 23-24 & $(29 \pm 10) \times 10^{-5}$ & & \\
\hline
\end{tabular}

Assuming $\left(Q_{\left.\mathrm{H}_{2} \mathrm{O}\right)} / Q_{\mathrm{OH}}\right)=1.1$.

b The range of production rates reflects calculations with different Haser parameters. The lower values (Schleicher 1997) use the Haser parameters of Cochran and Schleicher (1993). The larger ones are those computed with OH and OH-parent scalelengths at $r_{h}=1 \mathrm{AU}$ of $1.05 \times 10^{5}$ and $5.77 \times 10^{4} \mathrm{~km}$, respectively, and an $\mathrm{OH}$ expansion velocity of $0.75 \mathrm{~km} \mathrm{~s}{ }^{-1}$. These latter parameters assume that $\mathrm{A} 6 \mathrm{OH}$ is collisionally thermalized and follows the $\mathrm{H}_{2} \mathrm{O}$ outflow, as expected in the region sampled by the $\sim 0.5^{\prime}$ diameter aperture. 
TABLE 6

LINE INTENSITIES OF SOME HDO ROTATIONAL LINES.

\begin{tabular}{cccc}
\hline \hline Line & $\nu[\mathrm{GHz}]$ & \multicolumn{3}{c}{$\left.\int T_{B} d v[\mathrm{~K} \mathrm{~km} \mathrm{~s}]^{-1}\right]^{\mathrm{b}}$} \\
\cline { 3 - 4 } & & \multicolumn{1}{c}{$d=30 \mathrm{~m}^{\mathrm{c}}$} & \multicolumn{1}{c}{$d=10 \mathrm{~m}^{\mathrm{c}}$} \\
\hline & & & \\
$Q_{\mathrm{H}_{2} \mathrm{O}}=2 \times 10^{29} \mathrm{~s}^{-1}, r_{h}=1 A U, \Delta=0.1 A U$ \\
$1_{10^{-}} 1_{11}$ & 80.578 & $5.15(4.63,3.90,1.04) 10^{-2}$ & $1.94(1.38,1.28,0.34) 10^{-2}$ \\
$3_{12}-2_{21}$ & 225.897 & $6.03(6.06,5.98,0.05) 10^{-2}$ & $1.74(1.72,2.02,0.02) 10^{-2}$ \\
$2_{11}-2_{12}$ & 241.562 & $1.09(1.08,1.01,0.07) 10^{-1}$ & $3.75(3.53,3.42,0.24) 10^{-2}$ \\
$2_{20}-3_{13}$ & 266.161 & $4.14(4.07,5.63,0.02) 10^{-2}$ & $8.30(7.80,19.1,0.08) 10^{-3}$ \\
$1_{01}-0_{00}$ & 464.925 & $1.38(1.38,1.32,4.59)$ & $5.44(5.71,4.49,15.6) 10^{-1}$ \\
$3_{12}-3_{13}$ & 481.780 & $1.05(1.06,1.03,0.01) 10^{-1}$ & $3.44(3.45,3.52,0.03) 10^{-2}$ \\
$2_{02}-1_{11}$ & 490.597 & $3.63(3.64,3.46,1.47)$ & $1.35(1.38,1.16,0.49)$ \\
$1_{10}-1_{01}$ & 509.292 & $12.2(12.2,11.6,3.09)$ & $4.56(4.44,3.97,1.06)$ \\
$2_{11}-2_{02}$ & 599.927 & $12.8(12.8,12.4,0.87)$ & $4.59(4.55,4.24,0.30)$ \\
$Q_{\mathrm{H}_{2} \mathrm{O}}=5 \times 10^{30} s^{-1}, r_{h}=1 A U, \Delta=1 A U$ & \\
$1_{10}-1_{11}$ & 80.578 & $1.11(0.94,0.81,0.22) 10^{-1}$ & $2.96(2.01,2.02,0.54) 10^{-2}$ \\
$3_{12}-2_{21}$ & 225.897 & $1.31(1.32,1.39,0.01) 10^{-1}$ & $2.88(2.89,4.15,0.04) 10^{-2}$ \\
$2_{11}-2_{12}$ & 241.562 & $2.56(2.53,2.36,0.17) 10^{-1}$ & $7.09(6.65,7.11,0.50) 10^{-2}$ \\
$2_{20}-3_{13}$ & 266.161 & $7.63(7.42,13.3,0.05) 10^{-2}$ & $1.23(1.16,4.03,0.02) 10^{-2}$ \\
$1_{01}-0_{00}$ & 464.925 & $3.57(3.61,3.18,11.1)$ & $1.47(1.57,1.01,3.51)$ \\
$3_{12}-3_{13}$ & 481.780 & $2.51(2.53,2.50,0.02) 10^{-1}$ & $6.95(7.01,7.99,0.07) 10^{-2}$ \\
$2_{02}-1_{11}$ & 490.597 & $9.26(9.36,8.39,3.57)$ & $3.29(3.41,2.63,1.12)$ \\
$1_{10}-1_{01}$ & 509.292 & $30.7(30.5,28.2,7.52)$ & $10.9(10.2,9.05,2.41)$ \\
$2_{11}-2_{02}$ & 599.927 & $32.0(32.0,30.2,2.13)$ & $10.5(10.3,9.78,0.69)$ \\
\hline
\end{tabular}

${ }^{\mathrm{a}}$ Assuming a $(\mathrm{D} / \mathrm{H})_{\mathrm{H}_{2} \mathrm{O}}$ ratio of $25 \times 10^{-5}\left(\mathrm{Q}_{\mathrm{HDO}} / \mathrm{Q}_{\left.\mathrm{H}_{2} \mathrm{O}\right)}=50 \times 10^{-5}\right)$.

${ }^{b}$ Calculations are performed with the comprehensive model which includes collisions with electrons. The three values in parenthesis are those computed without electronic collisions, under LTE assumptions and at fluorescence equilibrium, respectively. The model parameters are $T_{k i n}=70 \mathrm{~K}, v_{e x p}=0.75 \mathrm{~km} \mathrm{~s}^{-1}$ and $\sigma_{c}=$ $5 \times 10^{-14} \mathrm{~cm}^{2}$.

${ }^{\mathrm{c}}$ Antenna diameter. 